Louisiana State University

LSU Digital Commons

Faculty Publications

Department of Physics \& Astronomy

3-10-2019

\title{
Supernova 2017eaw: Molecule and Dust Formation from Infrared Observations
}

\author{
Samaporn Tinyanont \\ California Institute of Technology \\ Mansi M. Kasliwal \\ California Institute of Technology \\ Kelsie Krafton \\ Louisiana State University \\ Ryan Lau \\ JAXA Institute of Space and Astronautical Science \\ Jeonghee Rho \\ SETI Institute
}

See next page for additional authors

Follow this and additional works at: https://digitalcommons.Isu.edu/physics_astronomy_pubs

\section{Recommended Citation}

Tinyanont, S., Kasliwal, M., Krafton, K., Lau, R., Rho, J., Leonard, D., De, K., Jencson, J., Mawet, D., MillarBlanchaer, M., Nilsson, R., Yan, L., Gehrz, R., Helou, G., Van Dyk, S., Serabyn, E., Fox, O., \& Clayton, G. (2019). Supernova 2017eaw: Molecule and Dust Formation from Infrared Observations. Astrophysical Journal, 873 (2) https://doi.org/10.3847/1538-4357/ab0897

This Article is brought to you for free and open access by the Department of Physics \& Astronomy at LSU Digital Commons. It has been accepted for inclusion in Faculty Publications by an authorized administrator of LSU Digital Commons. For more information, please contact ir@lsu.edu. 


\section{Authors}

Samaporn Tinyanont, Mansi M. Kasliwal, Kelsie Krafton, Ryan Lau, Jeonghee Rho, Douglas C. Leonard, Kishalay De, Jacob Jencson, Dimitri Mawet, Maxwell Millar-Blanchaer, Ricky Nilsson, Lin Yan, Robert D. Gehrz, George Helou, Schuyler D. Van Dyk, Eugene Serabyn, Ori D. Fox, and Geoffrey Clayton 


\title{
Supernova 2017eaw: Molecule and Dust Formation from Infrared Observations
}

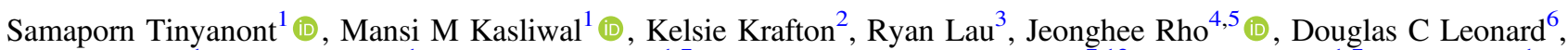 \\ Kishalay De ${ }^{1}$, Jacob Jencson ${ }^{1}$ (1), Dimitri Mawet ${ }^{1,7}$ (D), Maxwell Millar-Blanchaer ${ }^{7,12}$, Ricky Nilsson ${ }^{1,7}$ (D), Lin Yan ${ }^{1}$, \\ Robert D Gehrz $^{8}$, George Helou ${ }^{9}$, Schuyler D Van Dyk ${ }^{9}$ (i), Eugene Serabyn ${ }^{10}$, Ori D Fox ${ }^{11}$ (D), and Geoffrey Clayton ${ }^{2}$ (iD \\ ${ }^{1}$ Division of Physics, Mathematics and Astronomy, California Institute of Technology, 1200 E. California Blvd., Pasadena, CA 91125, USA; st@astro.caltech.edu \\ ${ }^{2}$ Department of Physics and Astronomy, Louisiana State University, Baton Rouge, LA 70803, USA \\ ${ }^{3}$ Institute of Space \& Astronautical Science, Japan Aerospace Exploration Agency, 3-1-1 Yoshinodai, Chuo-ku, Sagamihara, Kanagawa 252-5210, Japan \\ ${ }^{4}$ SETI Institute, 189 N. Bernardo Ave, Suite 200, Mountain View, CA 94043, USA \\ ${ }^{5}$ SOFIA Science Center, NASA Ames Research Center, MS 232, Moffett Field, CA 94035, USA \\ ${ }^{6}$ Department of Astronomy, San Diego State University, San Diego, CA 92182, USA \\ ${ }^{7}$ Jet Propulsion Laboratory, California Institute of Technology, Pasadena, CA 91109, USA \\ ${ }^{8}$ Minnesota Institute for Astrophysics, School of Physics and Astronomy, University of Minnesota, 116 Church Street, S.E., Minneapolis, MN 55455, USA \\ ${ }^{9}$ Caltech/IPAC, Mailcode 100-22, Pasadena, CA 91125, USA \\ ${ }^{10}$ Jet Propulsion Laboratory, California Institute of Technology, 4800 Oak Grove Dr, Pasadena, CA 91109, USA \\ ${ }^{11}$ Space Telescope Science Institute, 3700 San Martin Dr, Baltimore, MD 21218, USA \\ Received 2019 January 28; revised 2019 February 16; accepted 2019 February 18; published 2019 March 13
}

\begin{abstract}
We present infrared (IR) photometry and spectroscopy of the Type II-P SN 2017eaw and its progenitor in the nearby galaxy NGC 6946. Progenitor observations in the $K s$ band in four epochs from 1 yr to 1 day before the explosion reveal no significant variability in the progenitor star greater than $6 \%$ that lasts longer than 200 days. SN 2017eaw is a typical SN II-P with near-IR and mid-IR photometric evolution similar to those of SNe $2002 \mathrm{hh}$ and 2004et, other normal SNe II-P in the same galaxy. Spectroscopic monitoring during the plateau phase reveals a possible high-velocity He I $1.083 \mu \mathrm{m}$ absorption line, indicative of a shock interaction with the circumstellar medium. Spectra between 389 and 480 days postexplosion reveal a strong CO first overtone emission at 389 days, with a line profile matching that of SN 1987A from the same epoch, indicating $\sim 10^{-3} M_{\odot}$ of CO at $1800 \mathrm{~K}$. From the 389 days epoch until the most recent observation at 566 days, the first overtone feature fades while the $4.5 \mu \mathrm{m}$ excess, likely from the $\mathrm{CO}$ fundamental band, remains. This behavior indicates that the $\mathrm{CO}$ has not been destroyed, but that the gas has cooled enough that the levels responsible for first overtone emissions are no longer populated. Finally, the evolution of Spitzer $3.6 \mu$ m photometry shows evidence for dust formation in SN 2017eaw, with a dust mass of $10^{-6}$ or $10^{-4} M_{\odot}$ assuming carbonaceous or silicate grains, respectively.
\end{abstract}

Key words: circumstellar matter - supernovae: individual (SN2017eaw)

\section{Introduction}

Massive stars $\left(M \gtrsim 8 M_{\odot}\right)$ conclude their evolution in a core-collapse supernova (CCSN) when the nuclear fuel in their core is exhausted. The most common CCSNe are of Type II-P, whose defining features are a plateau in their light curves where the bolometric luminosity remains constant for $\sim 100$ days and strong hydrogen features in all phases of their spectral evolution (see, e.g., Filippenko 1997 for SN types). The persisting presence of hydrogen points to progenitor stars with hydrogen remaining in the stellar envelope at the time of core collapse. Archival pre-explosion observations of a number of nearby SNe II-P have indeed revealed hydrogen-rich red supergiant (RSG) progenitor stars (Smartt 2009 and references therein). While SNe II-P are the most common-and most well understood-among subtypes of CCSNe, there are several aspects of the explosion that remain to be understood, both theoretically and observationally. Examples of unanswered questions regarding SNe II-P include the landscape of the circumstellar medium (CSM) around their RSG progenitors and the chemical evolution and dust formation in their ejecta. Some SNe II-P show signatures of shock interaction with a CSM, even though the typical RSG progenitors are expected to have steady wind-driven mass loss with no dense CSM at the time of core collapse. These signs of shock interaction include

\footnotetext{
${ }^{12}$ Hubble Fellow.
}

weak X-ray and radio emission from the shocked material and infrared (IR) emission from dust, which is either preexisting or newly formed. Another aspect of SNe II-P that is not yet well understood is the chemical evolution of their ejecta. CCSNe are a promising source of dust in the early universe, making it crucial to understand how molecules and dust grains evolve in their ejecta. While chemical evolution models of the ejecta of $\mathrm{SNe}$ II-P have existed for decades, the observational data (mostly IR) required to test them remain sparse, due to the limited number of nearby normal SNe II-P that can be observed in great detail out to late times.

While we expect SNe II-P in a pristine environment in comparison to $\mathrm{SNe}$ with stripped-envelope progenitors, recent observations, especially in the IR, show several SNe II-P with weaker signs of interactions with a CSM likely ejected within decades to centuries before the SN. In the strongest case of interaction with a dense CSM, one can observe narrow recombination lines from the CSM gas photoionized by highenergy photons from the interaction region (spectral Type In; Schlegel 1990). For SNe II-P, however, the narrow lines are not present, and the main signatures of the interaction are X-ray and radio emissions coming from the shocked CSM gas, with lower luminosity indicating a less dense CSM in comparison to that of SNe IIn (e.g., SNe 2013ej, Chakraborti et al. 2013; 2011ja, Chakraborti et al. 2016). CSM interactions also leave spectroscopic imprints, such as a high-velocity absorption 
feature of $\mathrm{H} \alpha$ and $\mathrm{He} \mathrm{I} 1.083 \mu \mathrm{m}$ (Chugai et al. 2007) and asymmetric and multipeaked hydrogen lines associated with a toroidal CSM (e.g., SNe 2007od, Andrews et al. 2010; 2011ja, Andrews et al. 2016). Finally, the CSM interaction can trigger new dust formation and heat preexisting dust, both of which emit in the thermal IR.

The CSM interaction can trigger dust formation by generating a reverse shock propagating back into the ejecta, creating a cold dense shell (CDS) between the forward and reverse shocks. Conditions in the CDS are suitable for dust formation. In some $\mathrm{SNe}$, CDS dust formation can happen early ( $\lesssim 300$ days), before the outer ejecta have cooled enough for dust formation (although recently Sarangi et al. 2018a have argued that before 380 days, high-energy photons from the shock interaction region inhibit dust formation in the CDS). Early dust formation, likely in the CDS, has been observed in many interacting SNe, for example, SNe 2005ip (IIn Fox et al. 2009; Smith et al. 2009b), 2006od (II-P Andrews et al. 2010), 2010j1 (IIn Gall et al. 2014), and 2011ja (II-P Andrews et al. 2016; Tinyanont et al. 2016). Nearby SNe 2004dj and 2004et both showed rebrightening in their IR light curves attributable to dust formation (Kotak et al. 2009; Fabbri et al. 2011; Meikle et al. 2011; Szalai et al. 2011). In SN 2004et, the dust formation at $\sim 1000$ days postexplosion likely occurred in the CDS between the SN forward shock and the reverse shock generated by a delayed CSM interaction. Kotak et al. (2009) showed that the spectral energy distribution (SED) from 200 to 500 days postexplosion exhibited strong signatures of carbon monoxide (CO) and silicon monoxide (SiO) at 4.67 and $8 \mu \mathrm{m}$ along with a broad silicate grain feature around $9.7 \mu \mathrm{m}$, suggesting a mixture of carbonaceous and silicate grain formation. Most of these observations were enabled by Spitzer Space Telescope (Werner et al. 2004; Gehrz et al. 2007)/Infrared Array Camera (IRAC; Fazio et al. 2004) observations and, for a limited number of CCSNe, by the cold mission instruments Infrared Spectrograph (IRS; Houck et al. 2004) and the Multiband Imaging Photometer for Spitzer (MIPS; Rieke et al. 2004). These observations are sensitive to the thermal emission from dust with temperature of $200-1000 \mathrm{~K}$. We note here that the CSM dust can create an IR light echo from the peak SN light without any CSM-shock interaction. However, observations have shown that IR light echoes cannot explain the large amount of IR radiation seen at late times, even in SN 2011dh, where the IR light curve faded quickly in comparison to other CCSNe (Helou et al. 2013).

In addition to observations of a handful of $\mathrm{SNe}$ during its cold mission, Spitzer observed multiple $\mathrm{SNe}$ during the warm mission when only the IRAC 3.6 and $4.5 \mu \mathrm{m}$ imaging channels were available. While the full spectral coverage, especially around the $9.7 \mu \mathrm{m}$ silicate feature, is not available, warm Spitzer studies have shown a diversity in the IR light curve evolution among SNe II-P. Our previous work (Tinyanont et al. 2016) presented a Spitzer survey of 36 CCSNe in nearby galaxies out to $20 \mathrm{Mpc}$ showing that IR emission later than 100 days is common among CCSNe, with some SNe II-P showing signs of CSM interactions (see Szalai et al. 2018 for a more recent compilation of Spitzer observations of SNe). The most unique among our sample were SN 2011ja with bright and almost constant IR luminosity out to $\sim 1000$ days and SN 2013ej with IR rebrightening. Both SNe showed signs of CSM interactions from X-ray observations (Chakraborti et al. 2013, 2016), optical spectroscopy (Andrews et al. 2016;
Mauerhan et al. 2017), and, for SN 2013ej, spectropolarimetry (Mauerhan et al. 2017). SN 2011ja likely formed dust very early at 105 days postexplosion (Andrews et al. 2016). These observations demonstrate the range of epochs at which the CSM interactions or dust formation begin around SNe II-P.

Dust grains can form not only in the CDS created by the CSM interaction, but also in the ejecta of the SN itself after they have sufficient time to cool. This is arguably the more important channel of dust formation because it can operate whether or not the SN has a CSM interaction. To understand dust formation in the ejecta, it is crucial to have a realistic chemical evolution model. Several models in the past have relied on simplistic classical nucleation theory (CNT) or kinetic nucleation theory, in which dust formation is parameterized in some way to simplify the calculation (see Sarangi et al. 2018b; Sluder et al. 2018 for a summary of different classes of chemical evolution models). In the past decade, modelers have started to employ molecular nucleation theory (MNT), which simulates molecule and dust formation in the SN ejecta only using a realistic network of chemical reactions. The MNT models are the only ones that explicitly simulate molecule and dust evolution simultaneously. Molecules are crucial for dust formation because they are effective at cooling the ejecta to temperatures suitable for dust condensation and can act as seed nuclei. Some molecules are direct precursor species to dust grains. For example, $\mathrm{SiO}$ is a building block for silicate grains. The summary of observational data on $\mathrm{CO}$ and $\mathrm{SiO}$ mass evolution in a few $\mathrm{SNe}$ in comparison to a $15 M_{\odot}$ model can be found in Figures 3 and 4 in Sarangi \& Cherchneff (2013), respectively. The same comparison for observed dust mass in $12 \mathrm{SNe}$ and four different progenitor models can be found in Figure 10 from Sarangi \& Cherchneff (2015). The observations required to measure molecule and dust mass are difficult and are sparse as a result. For molecule formation in SN ejecta, only $\sim 10$ observations have been reported (see the summaries by Gerardy et al. 2002 and Sarangi et al. 2018b, and references therein). Additional observations are required to bridge the gap between the small dust masses of $10^{-4}-10^{-3} M_{\odot}$ inferred at a few hundred days postexplosion from near to mid-IR observations (e.g., Szalai \& Vinkó 2013; Tinyanont et al. 2016) and the larger dust masses of $0.01-0.1 M_{\odot}$ inferred in supernova remnants Cas A (Rho et al. 2008; Barlow et al. 2010), Crab Nebula (Gomez et al. 2012), Sgr A East (Lau et al. 2015), and SN 1987A (Indebetouw et al. 2014; Matsuura et al. 2015) inferred from far-IR and submillimeter observations.

SN 2017eaw is the most recent nearby event for which detailed late-time IR observations, required to investigate the issues described above, were possible. SN 2017eaw was discovered on 2017 May 14 in NGC 6946 as its 10th SN in the past century (Wiggins 2017). Early spectroscopic observations showed that it was a typical young SN II-P with a spectrum similar to that of SN 1999gi at 3.8 days postexplosion (Tomasella et al. 2017). Early radio observations immediately after detection resulted in nondetections at $1.39,5.1$, and $15 \mathrm{GHz}$ by the Giant Metrewave Radio Telescope, the electronic Multi-element Remotely Linked Interferometer Network, and the Arcminute Microkelvin Imager Large Array (AMI-LA), respectively. At $1.39 \mathrm{GHz}$, Nayana \& Chandra (2017a) reported an early nondetection on 2017 May 18 with an upper limit of $114 \mu \mathrm{Jy}$. Thirty-six days later on 2017 June 23, the SN was detected at $230 \pm 68 \mu \mathrm{Jy}$ (Nayana \& Chandra 2017b). At $5.1 \mathrm{GHz}$, Argo et al. (2017a) reported an early nondetection on 2018 May 20 


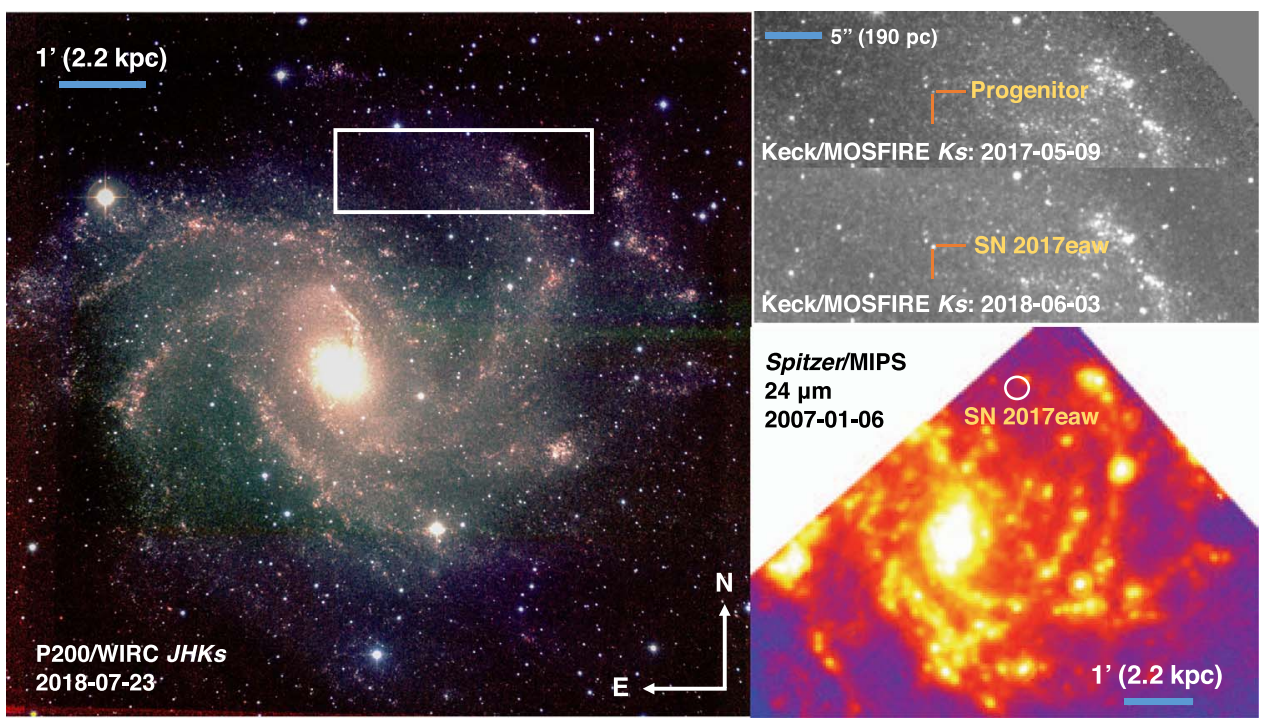

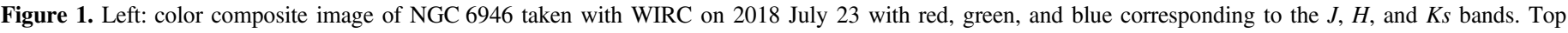

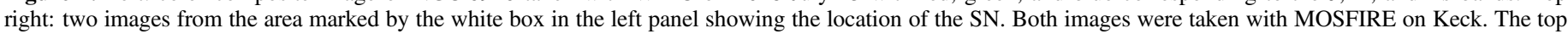

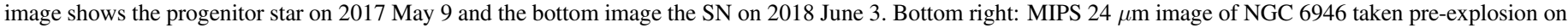

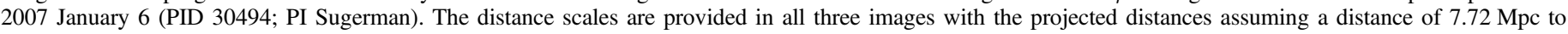
NGC 6946. The orientations are all north up and east to the left.

with an upper limit of $65 \mu \mathrm{Jy} /$ beam. Subsequent observations on May 29.55, 30.81, and June 1.71 detected a rising flux of $280 \pm 25,350 \pm 51$, and $427 \pm 23 \mu \mathrm{Jy}$, respectively (Argo et al. 2017b). Lastly, AMI-LA observations at $15 \mathrm{GHz}$ on May 15,17 , and 20 did not yield any detections with upper limits of 0.5-1 mJy (Bright et al. 2017; Mooley et al. 2017). No AMI-LA observations have been reported during the epochs in which the $\mathrm{SN}$ was detected at 1.39 and $5 \mathrm{GHz}$. This increasing radio flux may be a sign of the SN shock running into the denser part of the CSM. X-ray observations by Swift on May 14 (Kong \& Li 2017) and the Nuclear Spectroscopic Telescope Array (NuStar; Harrison et al. 2013) on May 21 (Grefensetette et al. 2017) revealed a rising X-ray flux, also indicative of CSM interactions. The NuStar observations showed a hard spectrum with photons detected up to $30 \mathrm{keV}$ and also revealed a shock-heated ionized Fe line at $6.65 \mathrm{keV}$. SN 2017eaw has also been observed with Spitzer/IRAC in the 3.6 and $4.5 \mu \mathrm{m}$ bands as part of the ongoing SPitzer InfraRed Intensive Transients Survey (SPIRITS; Kasliwal et al. 2017). In addition to these potential signs of CSM interactions, optical and near-IR observations of this SN are consistent with those of other SNe with early dust formation. Tsvetkov et al. (2018) published optical photometry of the SN until 200 days postexplosion along with a preliminary comparison to different light curve models. They noted the photometric similarity between this SN and SN 2004et, an aforementioned SN II-P in the same galaxy. Rho et al. (2018) reported Gemini nearIR spectroscopic follow-up from 22 to 205 days postdiscovery showing an emerging $\mathrm{CO}$ first overtone emission at $\sim 2.3 \mu \mathrm{m}$, a clear signature of $\mathrm{CO}$ formation. They also reported a rising red continuum in the $K$ band that could be coming from hot dust.

Here we present and discuss results from IR observations of SN 2017eaw and its progenitor. Section 2 summarizes archival near-IR imaging of the progenitor star and IR photometry and spectroscopy of the SN with both ground-based telescopes and Spitzer. In Section 2.1, we report near-IR photometry of the progenitor star of SN 2017eaw detected in ground-based imaging, including one epoch by the Keck/Multi-Object
Spectrometer For Infra-Red Exploration (MOSFIRE) on 2017 May 9, five days before the discovery of the SN. We present IR light curves and spectroscopy of the $\mathrm{SN}$ in Sections 2.3, 2.4. We analyze the data in Section 3 and discuss the progenitor nonvariability in the $K$ band (Section 3.1 ), the IR photometry in comparison to other SNe II-P (Section 3.2), and the SED (Section 3.3) and CO line profile and its evolution (Section 3.5, 3.6). A summary and conclusions are in Section 4.

\section{Observations}

\subsection{Progenitor Infrared Photometry}

NGC 6946 is a nearby galaxy $(d=7.72 \mathrm{Mpc}$; Anand et al. 2018), extremely prolific at producing SNe. Numerous preexplosion observations of SN2017eaw's site, both from the ground and space, are available partly as a result of efforts to follow the temporal evolution of SNe 2002hh, 2004et, and 2008S. The progenitor star has been detected in archival Hubble and Spitzer images, with an SED consistent with that of a dusty RSG star (Khan 2017; van Dyk et al. 2017; Kilpatrick \& Foley 2018). As part of the follow-up campaign for SPIRITS, we have imaged the SN site with the Wide field InfraRed Camera (WIRC; Wilson et al. 2003) on the 200 inch telescope at Palomar Observatory (P200 hereafter) in the $J, H$, and $K s$ bands on 2016 October 11, 7 months before the explosion, and in the Ks band on 2017 May 3, 11 days before the SN was first detected. We also imaged the SN site in the $K s$ band using MOSFIRE (McLean et al. 2012) at the Keck Observatory on 2016 May 30 and 2017 May 9. The second epoch was only 5 days before the SN was first detected. In both cases, data were taken with dithering patterns that send the galaxy in and out of the field of view to measure and subtract the sky background. Science images were dark-subtracted and flattened using a flat-field image obtained from median-combining dithered sky images with sources masked out.

First, to identify the progenitor star, we compare preexplosion images to an image of SN 2017eaw a year postexplosion. Figure 1 (left) shows NGC 6946 in near-IR 

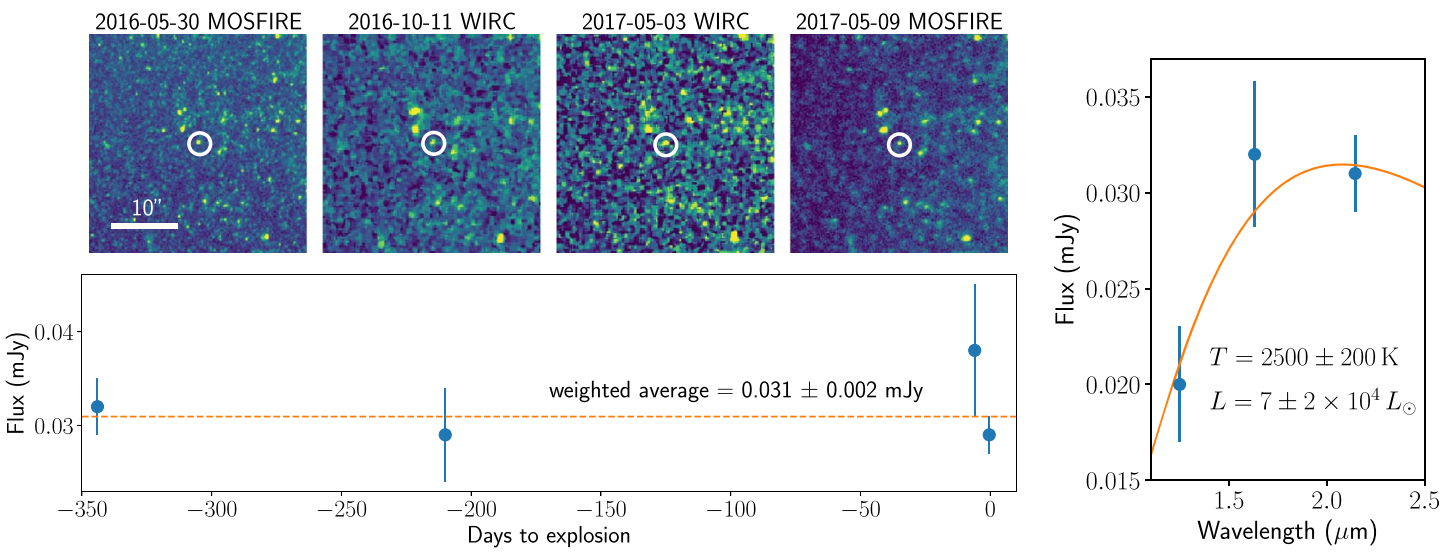

Figure 2. Left: images of the progenitor of SN 2017eaw from a year to $\sim 1$ day before explosion (5 days before first detection) taken in the Ks band with Keck/ MOSFIRE and P200/WIRC. The progenitor is encircled in each image. WIRC images have been smoothed using a median filter for visualization. Photometry from these images (shown below the images) revealed no significant variability of the progenitor at a $6 \%$ level. Right: near-IR SED of the progenitor star corrected for foreground extinction (Schlafly \& Finkbeiner 2011). Data in the $J$ and $H$ bands are from 2016 October 11, while the $K s$ band is the weighted average over all four epochs shown on the left. Assuming $d=7.72 \mathrm{Mpc}$, the best blackbody parameters fitted to the SED are $T=2500 \pm 200 \mathrm{~K}$ and $L=7 \pm 2 \times 10^{4} L \odot$. These parameters are consistent with an RSG progenitor.

Table 1

Near-infrared Photometry

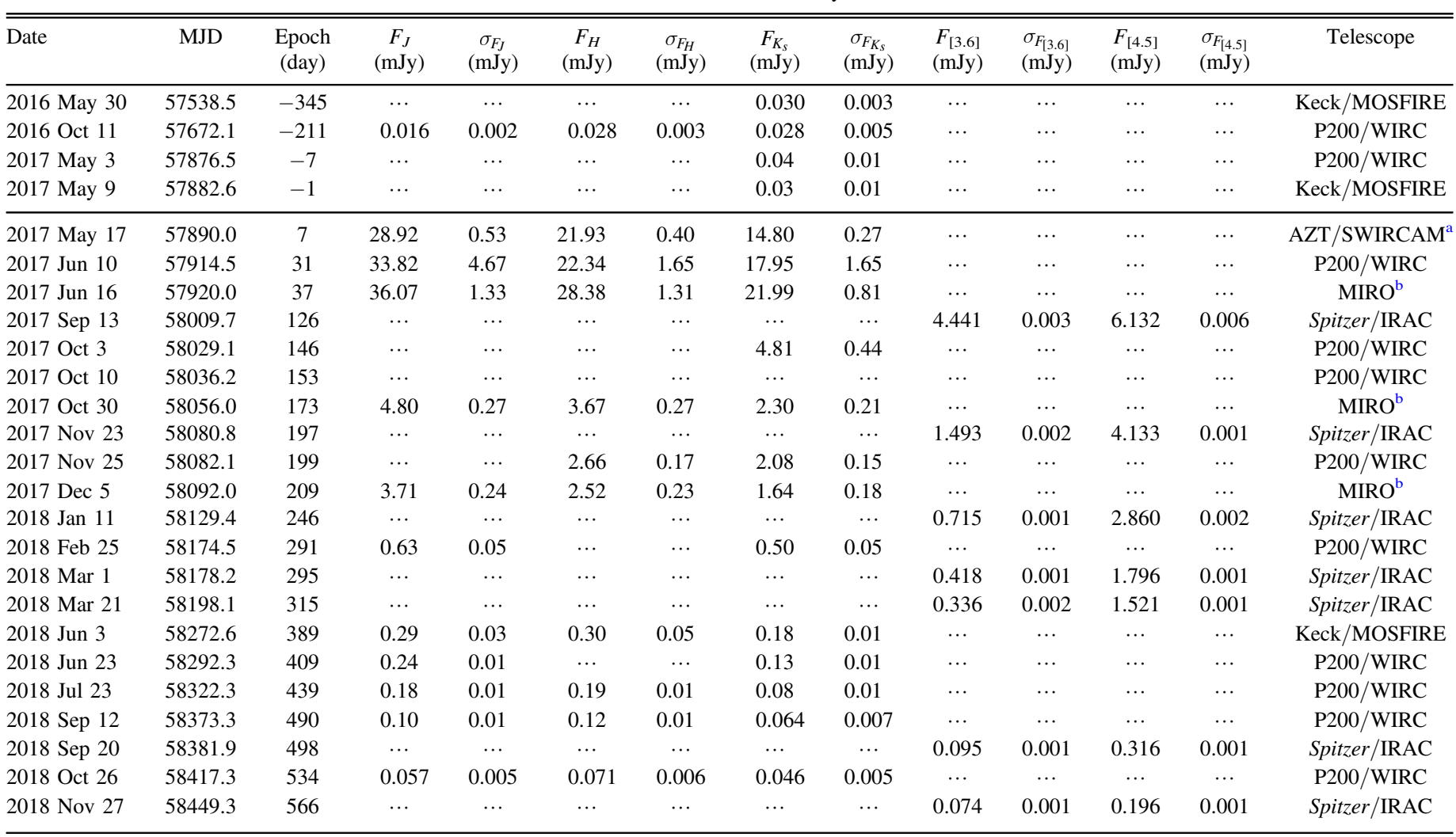

Notes.

a Arkharov et al. (2017) using the Short-Wave Infrared Camera (SWIRCAM) on the AZT-24 telescope at Campo Imperatore Observatory.

${ }^{\mathrm{b}}$ Rho et al. (2018) using Mount Abu Infrared Observatory (MIRO).

false color with the SN site annotated. The upper right panel shows the SN site (imaged by Keck/MOSFIRE in the $K s$ band) before and after the explosion to demonstrate that we have identified the progenitor star that is spatially coincident with the SN. We also note from these images that the galaxy light at the SN location is low in these bands, and we did not attempt any galaxy light subtraction. Moreover, the lower right panel of Figure 1 shows a $24 \mu \mathrm{m}$ Spitzer/MIPS image showing that the dust emission from the $\mathrm{SN}$ location is also minimal. As such, we assume only Galactic extinction for SN 2017eaw $(E(B-V)=0.305$; Schlafly \& Finkbeiner 2011) with no host extinction (consistent with Tomasella et al. 2017).

A point source is detected at the location of the $\mathrm{SN}$ in all observations, as shown in Figure 2 top left. Aperture photometry with sky annulus subtraction was obtained with the zero-point determined using $\sim 100$ stars in the field of view with magnitudes 
Table 2

Properties of SNe II-P Used to Compare with SN 2017eaw

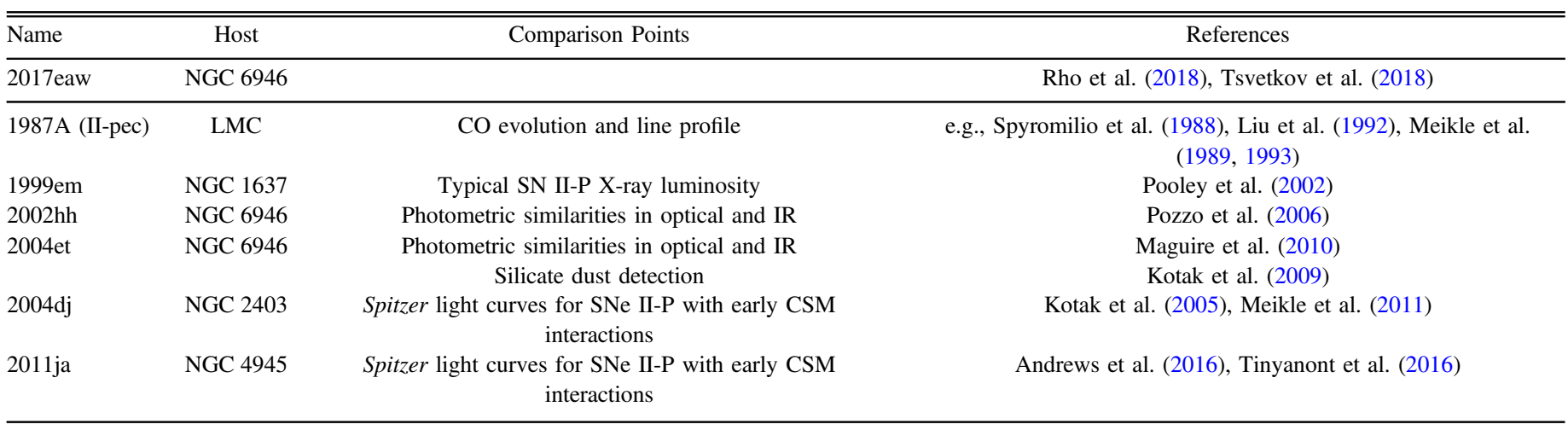

from the Two Micron All-Sky Survey (2MASS; Milligan et al. 1996; Skrutskie et al. 2006). The results are listed in Table 1 and plotted in Figure 2 (bottom left). We did not detect any significant variability, with a timescale greater than 200 days, of the progenitor star in the $K s$ band.

\subsection{Explosion Epoch}

Tsvetkov et al. (2018) published optical photometry in the UBVRI bands with data spanning from discovery (2018 May 14) until 208 days after discovery. They fitted a suite of light curve models to the data and derived an explosion date of 2017 May 4, 10 days before the first detection. We note that this is inconsistent with our progenitor observation on 2017 May 9, which showed no increase in flux from the progenitor at that epoch. We instead fitted a low-order polynomial to their $R$ - and $I$-band light curves, which best capture the rise. We constrain the explosion date to 2017 May 10, consistent with our progenitor observation and also with the spectroscopic age constraint (+3.8 days on May 14; Tomasella et al. 2017). This simple polynomial fitting neglects the shock break-out phase, which typically only lasts $\sim 1$ day. We take 2017 May 10 as the explosion date of SN 2017eaw throughout this paper.

\subsection{Supernova Photometry}

We obtained photometry of SN 2017eaw in the near-IR $J, H$, and $K s$ bands using P200/WIRC and Keck/MOSFIRE in nine epochs, spanning 31-490 days postexplosion, with seven epochs during the nebular phase. The process for data reduction and aperture photometry was the same as in the last section. All near-IR photometry results are presented in Table 1, including those reported in Arkharov et al. (2017) and Rho et al. (2018). The 2MASS magnitudes were converted to flux densities using zero-magnitude flux densities of 1594, 1024, and $666.7 \mathrm{Jy}$ for the $J, H$, and $K s$ bands, respectively (Cohen et al. 2003).

SN 2017eaw was observed with the Spitzer IRAC (Fazio et al. 2004) at $3.6 \mu \mathrm{m}$ and $4.5 \mu \mathrm{m}$ in six epochs: $126,197,246$, 295, 315, 498, and 566 days postexplosion (PID 13053, PI Kasliwal; PID 13239, PI Krafton). We used a stack of archival pre-explosion Spitzer images to estimate and remove the galaxy background and nearby source contamination. ${ }^{13}$ Archival images were rotated and aligned based on the sky coordinates

\footnotetext{
13 Archival Spitzer images used for background subtraction came from the following PIDs and PIs: 60071, 70008, 80131, 90178, PI Andrews; 80015, 10081, 11084, PI Kochanek; 10136, 11063, 80196, 13053, PI Kasliwal; 10002 , PI Sugerman.
}

supplied in Spitzer data, then median combined. In IRAC images in both channels of the 126 days epoch and the $3.6 \mu \mathrm{m}$ channel of the 215 days epoch, columns of low counts due to a saturating star in the field of view crossed the SN. To remove these low count columns, we fitted a Gaussian profile across each column and added the missing flux back in. We conducted aperture photometry on the background-subtracted images and applied appropriate aperture corrections as given by the IRAC instrument handbook. Spitzer photometry results are listed in Table 1. Spitzer fluxes were converted to magnitudes for plotting purposes (Figure 3) using the zero-magnitude fluxes of 280.9 and $179.7 \mathrm{Jy}$ for the 3.6 and $4.5 \mu \mathrm{m}$ channels, respectively. Figure 3 shows the near-IR light curves of SN 2017eaw in comparison with those of other well-studied SNe II-P. The top panel shows light curves of the $J, H, K s, 3.6$, and $4.5 \mu \mathrm{m}$ bands of SN 2017eaw in comparison to those of SNe II-P 2002hh and 2004et in the same galaxy. The bottom panel shows only Spitzer data points compared with all SNe IIP observed with Spitzer (photometry taken from Szalai et al. 2018). Throughout the paper, we assume the distance to NGC 6946 of $7.72 \mathrm{Mpc}$ based on the "tip of the red giant branch" technique (Anand et al. 2018, also used by Rho et al. 2018).

\subsection{Spectroscopy}

We obtained medium-resolution $(R \sim 2500)$ near-IR (1-2.5 $\mu \mathrm{m} ;$ YJHK) spectroscopy with P200/TripleSpec (Herter et al. 2008) on 2017 August 9 (91 days), 2017 September 3 (116 days), 2018 June 22 (408 days), and 2018 July 23 (439 days). TripleSpec is a long-slit $\left(1^{\prime \prime} \times 30^{\prime \prime}\right)$ spectrograph, and we observed the SN using an ABBA dither pattern for sky subtraction. Type A0V standard stars HIP 94140 and HIP 75230 were observed either immediately before or after the SN observations to provide telluric correction and flux calibration. Standard and SN observations were taken on different parts of the slit when the SN was observed after the standard to avoid persistence on the detector. The reduction for TripleSpec data was done using a version of Spextool modified for TripleSpec (Cushing et al. 2004). It applies field flattening, retrieves a wavelength solution from sky lines present in science observations, subtracts each $A B$ pair to remove most of the sky emission, and then fits a low-order polynomial to the different orders of spectral traces in the images. Simple spectral extraction is performed on the subtracted images (the optimal extraction algorithm (Horne 1986) is not available in this version of the software). Telluric 

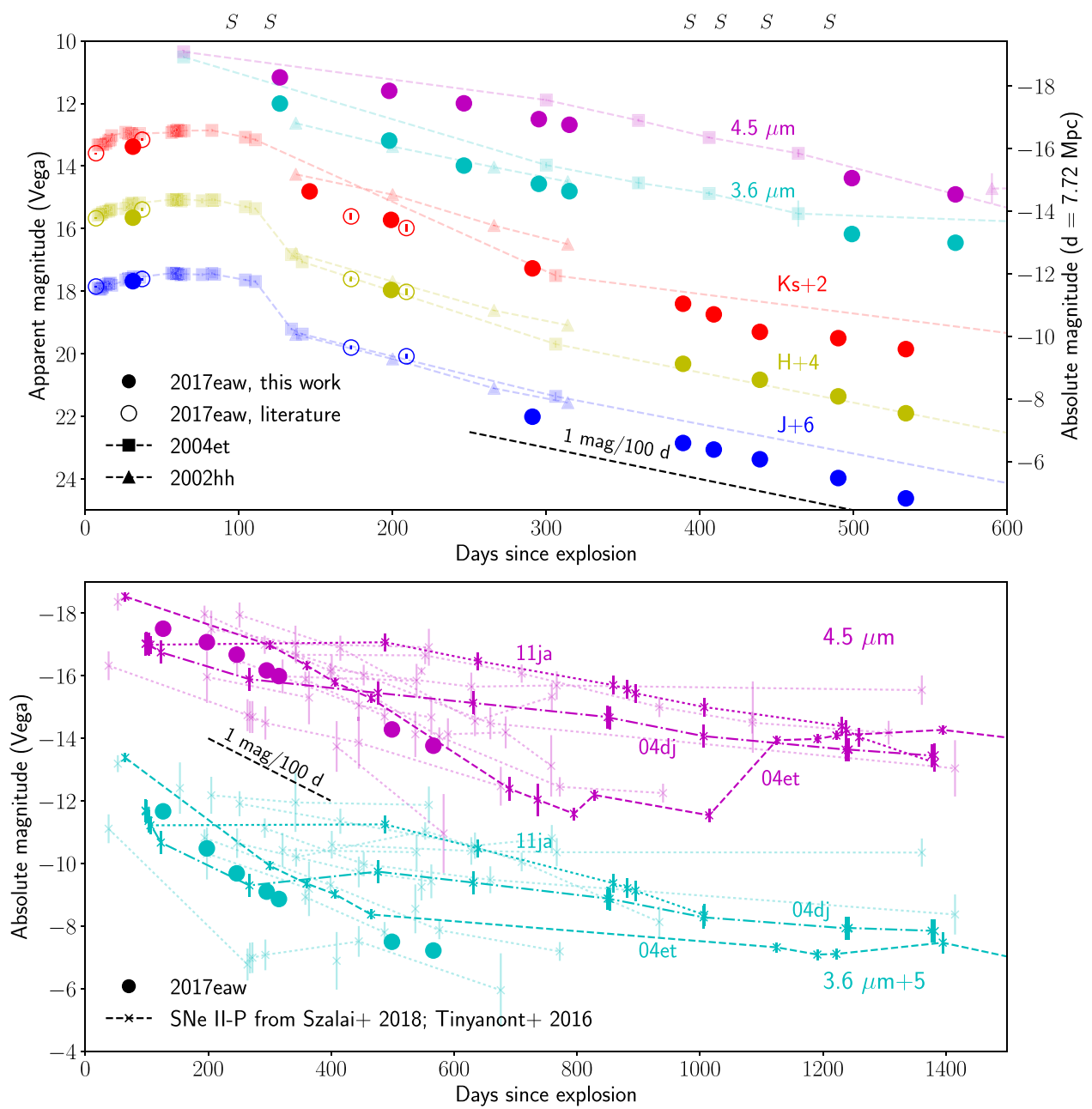

Figure 3. Top: near-IR photometry of SN 2017eaw in the $J, H$, and $K s$ bands and the IRAC 3.6 and $4.5 \mu$ m bands compared with photometry of SN 2004 et and SN 2002hh. Filled symbols for SN 2017eaw are our data, while open symbols are from Arkharov et al. (2017) and Rho et al. (2018). The JHKs photometry results for SN 2004et are from Maguire et al. (2010). Spitzer photometry results are from Fabbri et al. (2011). SN 2002hh data are from Pozzo et al. (2006). Note that the 3.6 $\mu \mathrm{m}$ data for this SN are from $L^{\prime}$-band ground-based observations. The "S" marks above the axis indicate epochs for which we obtained spectroscopy. Bottom: SN 2017eaw Spitzer photometry compared to all other SNe II-P photometry as aggregated by Szalai et al. (2018). No offsets between SNe are applied here, but all $3.6 \mu \mathrm{m}$ magnitudes are shifted by 5 mags for visualization. SNe 2004dj, 2004et, and 2011ja are highlighted for comparison. All magnitudes here are in the Vega system. The $1 \mathrm{mag} / 100$ days decline rate expected from light curves powered by radioactive decay of ${ }^{56}$ Co is plotted in both subplots.

and flux calibrations were performed using xtellcor (Vacca et al. 2003), which derives the instrument's efficiency by comparing the observed standard star spectrum with an A0V spectrum model from a high-resolution spectrum of Vega. We obtained $J$ - and $K$-band spectra with Keck/ MOSFIRE on 2018 June 3 (389 days). Data reduction and spectral extraction were performed using MOSFIRE's data reduction pipeline, ${ }^{14}$ and telluric and flux calibrations were performed using xtellcor. Finally, we obtained a $1-2.5 \mu \mathrm{m}$ spectrum using the Near-Infrared Echellette Spectrometer $(\text { NIRES })^{15}$ on the Keck telescope on 2018 September 2 (480 days). The instrument is very similar to TripleSpec, except with a narrower 0 ". 5 slit to take advantage of the better seeing from Maunakea. The observation strategy was similar. The data were reduced using a version of Spextool modified for NIRES, and the telluric and flux calibrations were performed using xtellcor. The standard spectra used for this calibration were from HIP 94140, observed before the SN. Figure 4

\footnotetext{
14 https://keck-datareductionpipelines.github.io/MosfireDRP/

15 https://www2.keck.hawaii.edu/inst/nires/
}

displays our near-IR spectra for SN 2017eaw from 91 to 480 days postexplosion with identifications of strong lines. The identifications were guided by Rho et al. (2018) and Meikle et al. (1993). Each epoch is multiplied by a factor listed on the right of the figure for visualization. TripleSpec spectra at 408 and 439 days are smoothed by a running median with a sevenpixel window, and the NIRES spectrum at 480 days is smoothed in the same way with a three-pixel window. The unsmoothed versions of the spectra are plotted as translucent lines.

\section{Analysis and Discussion}

\subsection{Progenitor Nonvariability and SED}

Some SNe II-P have shown stronger signs of CSM interaction than that of a typical SN II-P. This is indicative of a denser CSM in comparison to what is expected from steadystate RSG wind-driven mass loss. The required enhanced mass loss would result in a variability in the progenitor's light curve, which may be a gradual brightening over timescales of years, or short-term variability in the case of eruptive mass loss. Such 


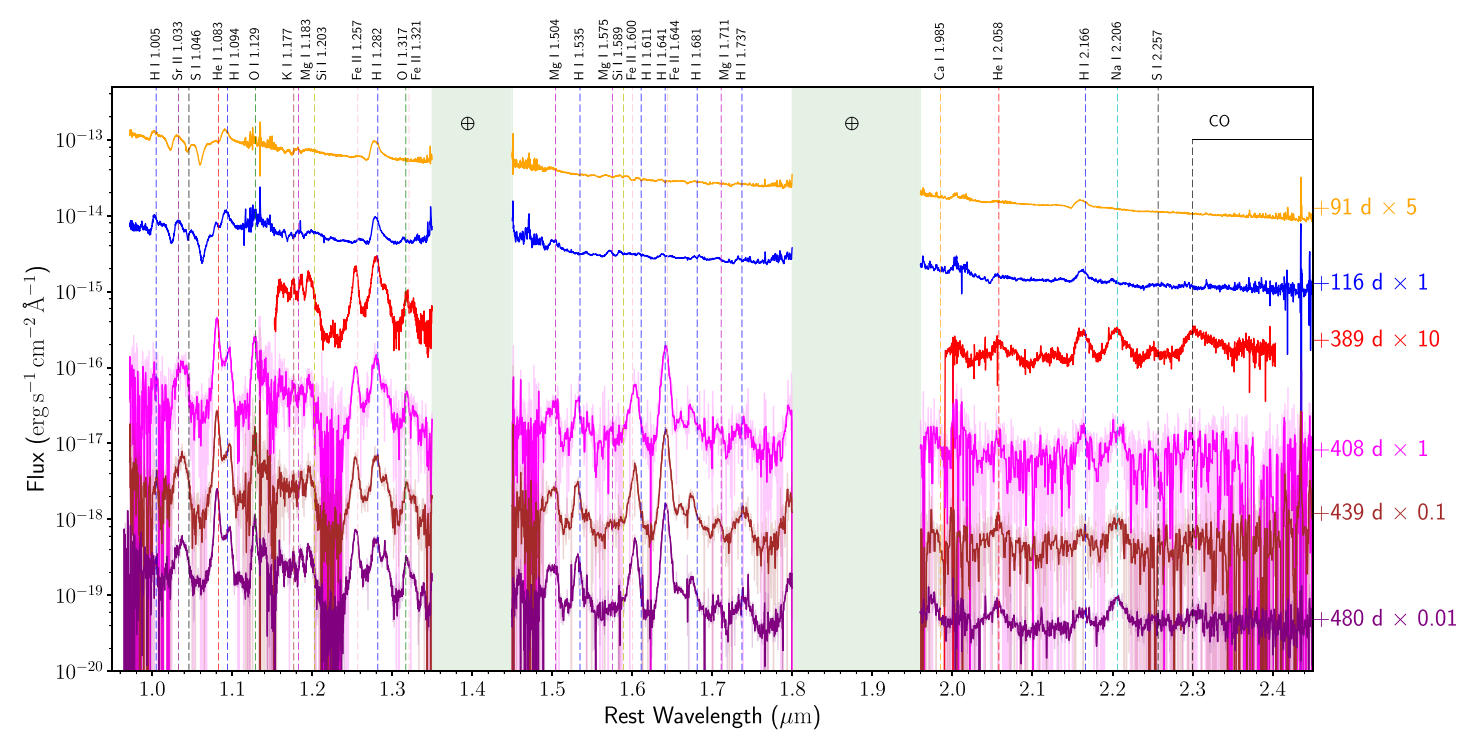

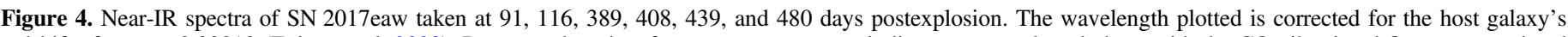

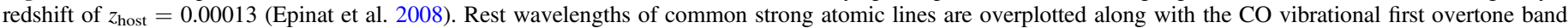

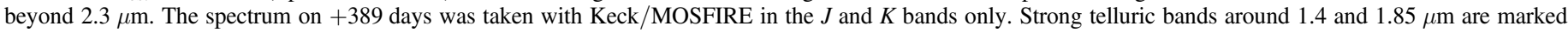

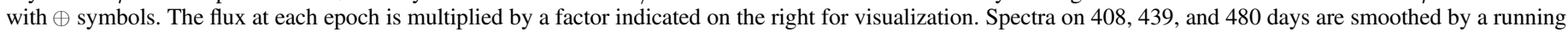
median with seven-, seven-, and three-pixel windows, respectively. Unsmoothed spectra are shown as translucent lines.

nearby and recently ejected CSM has been inferred in a variety of ways for a number of SNe II-P. Some SNe show signs of early dust formation and intermediate-velocity (few $\times 10^{3} \mathrm{~km} \mathrm{~s}^{-1}$ ) hydrogen emission lines or X-rays from the shocked CSM gas, for example, SNe 2007od (Andrews et al. 2010), 2011ja (Chakraborti et al. 2013; Andrews et al. 2016), and 2013ej (Chakraborti et al. 2016; Mauerhan et al. 2017). Some, like SN 2009kf, show early, strong ultraviolet emissions that require either a very energetic explosion or some degree of CSM interaction (Botticella et al. 2010; Moriya et al. 2011). Lastly, an emerging class of SNe II-P with very early spectra show narrow emission lines coming from the nearby CSM being ionized by the ultraviolet flash of the shock breakout, for example, SNe 2013fs (Yaron et al. 2017) and 2016bkv (Hosseinzadeh et al. 2018). These narrow lines disappear quickly (as opposed to $\mathrm{SNe}$ IIn with narrow emission lines near peak).

There are multiple proposed mechanisms for ejecting mass from an RSG within a decade pre-explosion, such as wavedriven mass loss (Fuller 2017; Fuller \& Ro 2018) and heavy element nuclear-burning instability $\left(9-11 M_{\odot}\right.$ RSG, Woosley \& Heger 2015). In the wave-driven scenario for RSGs, the predicted variability for the smallest eruption case is of order $20 \%$, ejecting $\sim 0.1 M_{\odot}$ (the lowest heating efficiency $(\eta=$ $1 / 3$ ) case in Fuller 2017). For the heavy element burning instability scenario, Smith \& Arnett (2014) argued that a 9-11 $M_{\odot}$ RSG can exhibit a detectable flash when the silicon burning commences. Nevertheless, the variability of the RSG progenitor star itself, which accompanies the mass loss event, has yet to be directly observed for progenitors to SNe II-P. ${ }^{16}$ In addition, there are other mechanisms proposed to explain the early-time observations that do not involve any dense CSM surrounding the progenitor (e.g., Kochanek 2018).

\footnotetext{
${ }^{16}$ Pre-SN eruption has been documented in some SNe Ibn/IIn, which have denser CSMs. The most well studied cases are SNe 2006jc (e.g., Foley et al. 2007; Pastorello et al. 2008) and 2009ip (e.g., Mauerhan et al. 2013). The definitive class of progenitors to those strongly interacting SNe has yet to be identified.
}

Indeed, our progenitor photometry in the near-IR (Figure 2 bottom left) shows that SN 2017eaw's progenitor is not variable in the $K s$ band from one year to one day pre-explosion on a timescale greater than 200 days. To put an upper limit on the variability of the progenitor, we compute the uncertainty of the weighted mean flux from the progenitor's $K s$-band photometry presented in Table 1 . The uncertainty of the mean is $0.25 \sqrt{\Delta F_{K_{s}}=\Sigma_{i}\left(\sigma_{F_{K_{s}},}^{2}\right)}$, where $i$ 's are four epochs at 345, 211, 7, and 1 days pre-explosion. The weighted-mean flux with the uncertainty is $\bar{F}_{K_{s}}=0.031 \pm 0.002 \mathrm{mJy}$. Assuming the distance of $7.72 \mathrm{Mpc}$ to the $\mathrm{SN}$, we obtain the variability limit of $\Delta \nu L_{\nu} \lesssim 6 \times 10^{3} L_{\odot}$. The total luminosity is $\nu L_{\nu}=$ $8 \times 10^{4} \tilde{L}_{\odot}$, typical of an RSG. This is consistent with the luminosity derived from fitting the SED, discussed later in this section. This corresponds to the variability upper limit of $6 \%$ over a year. In comparison, the weakest variability presented in Fuller (2017) is of order of 20\%. Further, short-term variability immediately before core collapse is ruled out by our last two epochs of observations within a few days before the explosion. We note that our finding of $K s$-band nonvariability does not conflict with that of Kilpatrick \& Foley (2018), who presented a $20 \%$ increase in the $4.5 \mu \mathrm{m}$ flux over $3 \mathrm{yr}$ pre-explosion, but no variability in the $3.6 \mu \mathrm{m}$ band.

The near-IR SED of the progenitor star in the near-IR is shown in Figure 2 (right). The photometry has been corrected for Galactic extinction $(E(B-V)=0.304$; Schlafly \& Finkbeiner 2011) with no additional host extinction. We fitted a blackbody curve to the SED and found the best-fit parameters to be $T=2500 \pm 200 \mathrm{~K}$ and $L=7 \pm 2 \times 10^{4} L_{\odot}$. We note that the blackbody luminosity is consistent with $\nu L_{\nu}$ obtained from $K s$-band observations presented above. These parameters are consistent with the progenitor star of SN 2017eaw being an RSG.

The progenitor star of SN 2017eaw has also been observed by the Large Binocular Telescope search for failed $\mathrm{SNe}$ in the optical ( $U B V R$ bands) in 35 epochs from $9 \mathrm{yr}$ to a few months before the explosion (Johnson et al. 2018). They found no significant stochastic variability in the luminosity down to 
$\Delta \nu L_{\nu} \lesssim 700 L_{\odot}$ in the $V$ and $R$ bands. Johnson et al. (2018) also reported nonvariability in three other progenitor stars to SNe 2013am, SN 2013ej, and ASASSN-2016fq. One of these SNe, SN 2013ej, has a nearby dense CSM inferred from X-ray (Chakraborti et al. 2016) and optical spectropolarimetry (Mauerhan et al. 2017) despite its progenitor having exhibited no variability in the last $5 \mathrm{yr}$ before the explosion. They argued that while outbursts may happen on a timescale shorter than their observing cadence, it is unlikely that the decline from these outbursts is fast enough to escape a detection because the dynamical and thermal timescales for these RSGs are much longer (years) than the observational cadence (months).

Our observations, along with those of Johnson et al. (2018), do not find major eruptive mass-loss events around SN 2017eaw's progenitor in the last $10 \mathrm{yr}$ of its life. The CSM around SN 2017eaw, inferred from radio and X-ray observations (Grefensetette et al. 2017; Kong \& Li 2017; Nayana \& Chandra 2017b), may be ejected in minor mass-loss events that cause variability smaller than $6 \%$ or last shorter than our observational cadence. Alternatively, the CSM around the RSG progenitor may be more akin to the compact CSM shell observed around Betelgeuse (Le Bertre et al. 2012). Mackey et al. (2014) presented a scenario in which such a compact CSM shell is constructed by a progenitor wind being trapped by ionizing photons in the star's environment. The CSM shell will eventually interact with the SN shock, and as shown by Smith et al. (2009a), this interaction will not be strong enough to produce typical Type IIn narrow lines. However, weak signs of CSM interactions may be detected in the X-ray and radio. For comparison, Pooley et al. (2002) derived from 0.2 to $10 \mathrm{keV}$ X-ray observations that SN 1999em (typical II-P) has a progenitor mass-loss rate of $\dot{M} \sim 2 \times 10^{-6} M_{\odot} \mathrm{yr}^{-1}$, similar to that of Betelgeuse. From the literature, the $0.3-10 \mathrm{keV}$ X-ray luminosity of SN 2017eaw is $1.1 \times 10^{39} \mathrm{erg} \mathrm{s}^{-1}$ at 11 days postexplosion (Grefensetette et al. 2017). In comparison, SN 1999em's X-ray luminosity is $2 \times 10^{38} \mathrm{erg} \mathrm{s}^{-1}$ at 4 days postexplosion (Pooley et al. 2002). Since the X-ray luminosity scales with $\dot{M}^{2}$ (see Equation (3.10) from Fransson et al. 1996), SN 2017eaw's progenitor would have a mass-loss rate of $\dot{M} \sim 5 \times 10^{-6} M_{\odot} \mathrm{yr}^{-1}$, which is in the typical range for an RSG. This mass-loss rate is consistent with the figure of $9 \times 10^{-7} M_{\odot} \mathrm{yr}^{-1}$ derived by Kilpatrick \& Foley (2018) using Hubble and Spitzer photometry. Hence, it is possible that the CSM for SN 2017eaw is created by a trapped wind like that seen around Betelgeuse, and not by eruptive mass loss in the last decade of the progenitor's life.

\subsection{Photometric Evolution and Comparison}

In order to assess SN 2017eaw's place in the Type II-P population, we compare its IR photometric evolution to those of other well-studied SNe II-P. Table 2 summarizes all SNe we used to compare to SN 2017eaw. We first caution that SN evolution in the near-IR remains poorly sampled in the nebular phase, making direct comparison to other individual $\mathrm{SNe}$ difficult. In the optical, Tsvetkov et al. (2018) has shown that SN 2017eaw bears photometric similarities to SN 2004et both in terms of flux and color evolution. Figure 3 (top) shows SN 2017eaw's 1-5 $\mu \mathrm{m}$ light curves in comparison to those of SN 2004et (Kotak et al. 2009; Maguire et al. 2010; Fabbri et al. 2011) and SN 2002hh (Pozzo et al. 2006), two normal SNe II-P in NGC 6946. SN 2017eaw is fainter than SN 2004et by about 0.5 mag in all bands, including the optical (Tsvetkov et al. 2018), without a strong wavelength dependence, which indicates that SN 2017eaw is intrinsically less energetic and not that it suffers more extinction and reddening. In Spitzer 3.6 and $4.5 \mu \mathrm{m}$ bands, the evolutions of SNe 2017eaw and 2004et are very similar. Except for the $4.5 \mu \mathrm{m}$ band, other near-IR bands show a similar linear decline of $\sim 1.5 \mathrm{mag} / 100$ days after 120 days. The $4.5 \mu \mathrm{m}$ band, however, declines at a slower rate of $0.80 \mathrm{mag} / 100$ days. For SN 2004et, Kotak et al. (2009) showed, using a series of SEDs with all Spitzer bands from 3.6 to $24 \mu \mathrm{m}$, that this different decline rate in the $4.5 \mu \mathrm{m}$ band is due to the $\mathrm{CO}$ fundamental vibrational emission at $4.65 \mu \mathrm{m}$. From this comparison alone, we can infer that CO is forming in SN 2017eaw. This is consistent with the emerging $\mathrm{CO}$ first overtone emission band starting around 120 days reported by Rho et al. (2018). Our spectra, presented in Section 2.4 and discussed in Section 3.5, also confirm the presence of CO. After 250 days, the decline rate in the $J, H$, and $K s$ bands is $1 \mathrm{mag} / 100$ days, the canonical decline rate for a light curve powered by ${ }^{56} \mathrm{Co}$ decay.

Figure 3 (bottom) shows SN 2017eaw's Spitzer light curves in comparison to all other SNe II-P light curves observed by Spitzer, aggregated by Szalai et al. (2018). Data here include photometry from Tinyanont et al. (2016). SN 2017eaw falls in the middle of the distribution of SNe II-P absolute magnitudes in both the 3.6 and $4.5 \mu \mathrm{m}$ bands, suggesting that it is a typical SN II-P. That SN 2017eaw is typical implies that the results we derive for SN 2017eaw in this paper may be more generally applicable to other SNe II-P that are fainter and more difficult to observe. More specifically, SN 2017eaw's (and SN 2004et's) decline rates of 1.5 and $0.8 \mathrm{mag} / 100$ days in the 3.6 and $4.5 \mu \mathrm{m}$ bands are typical among other SNe II-P with observations between 100 and 300 days. This suggests that strong $\mathrm{CO}$ emission in the $4.5 \mu \mathrm{m}$ band may be ubiquitous among SNe II-P. The implication here is that previous studies of SN dust based on warm Spitzer data with only 3.6 and $4.5 \mu \mathrm{m}$ data may provide unreliable dust estimates because the $4.5 \mu \mathrm{m}$ band is dominated not by thermal emission from dust, but CO line emission. The result is that the dust luminosityand, consequently, dust mass-is overestimated.

While SN 2017eaw's early temporal evolution is similar to that of most other SNe II-P, we note that it is markedly different from SNe 2004dj (Kotak et al. 2005) and 2011ja (Andrews et al. 2016; Tinyanont et al. 2016). Both of these $\mathrm{SNe}$ show signs of CSM interaction and dust formation at much earlier epochs: 65-165 days for SN 2004dj (Meikle et al. 2011) and 105 days for SN 2011ja (Andrews et al. 2016). Lastly, SN 2017eaw's resemblance to SN 2004et's Spitzer light curve presents an intriguing possibility that SN 2017eaw will rebrighten just like SN 2004et at $\sim 1000$ days because of shock interaction with a distant CSM shell (see Figure 3 bottom and Kotak et al. 2009). This possibility warrants continued monitoring of this SN, and other nearby CCSNe in the future, in the IR by either Spitzer or ground-based instruments. More generally, future IR observations of nearby CCSNe will reveal a range of epochs when CSM interactions or dust formation commence, from $\sim 100$ to $\sim 1000$ days postexplosion. Such observations will provide clues to the physical processes in the last stage of RSG evolution that are responsible for the distant CSM shell seen in, for example, SN 2004et.

\subsection{SED Modeling}

We performed radiative transfer (RT) modeling of the SEDs for SN 2017eaw to estimate the mass of dust associated with 
the SN. We used MOnte CArlo SimulationS of Ionized Nebulae (MOCASSIN; version 2.02.72), which is a fully selfconsistent 3D Cartesian dust RT code (Ercolano et al. 2003, 2005, 2008). The models accept multiple user inputs, but due to the limited wavelength coverage in our SED data, we limited the number of parameters we fitted, in order to avoid overfitting. While MOCASSIN takes a series of user inputs, such as number of dimensions, grid size, dust density, composition, and distribution, the only parameters we varied to fit the SED were the inner and outer radii of the dust shell, and the density at the inner edge of the shell. We discuss the other parameters, which are held constant, later in this section. The luminosity and temperature of the photon source (the $\mathrm{SN}$ ) were measured or extrapolated from the optical data. Any interactions, whether absorption or scattering, between photons and dust grains are governed by Mie scattering theory. MOCASSIN returns the temperature, mass, and opacity of the dust shells.

We used MOCASSIN to calculate the mass of dust at 126 , 197, 295, and 498 days, epochs for which we had Spitzer coverage. The optical data for 126 and 197 days are from Tsvetkov et al. (2018), while the 295 days epoch is extrapolated (plotted as open symbols). We checked that the extrapolated photometry did not differ significantly from SN 2004et photometry at the same epoch. We did not extrapolate to 498 days and simply used the same blackbody input for the 295 and 498 days epochs. The near-IR data for 126 days are from the flux-calibrated TripleSpec spectrum. Optical through near-IR photometry has been corrected for Galactic extinction $(E(B-V)=0.304$; Schlafly \& Finkbeiner 2011), assuming that the host galaxy extinction is negligible.

We chose to model this system as a central point source surrounded by a gas-free dust shell. The shell was further assumed to be "smooth," which means that there are no inhomogeneities ("clumps"), with the dust density profile falling by $r^{-2}$ from the inner radius $\left(R_{\text {in }}\right)$ to the outer radius $\left(R_{\text {out }}\right)$. We used the standard Mathis Rumpl Nordsieck (Mathis et al. 1977) power-law distribution, $a^{-3.5}$, to specify the size distribution of the dust grains. We tested two compositions for the dust grains: $100 \%$ amorphous carbon (amC) and 100\% silicate grains (Hanner 1988; Ossenkopf et al. 1992, respectively). We were unable to constrain the composition because of a lack of data beyond $4.5 \mu \mathrm{m}$. We do not fit the $R, I$, and $4.5 \mu \mathrm{m}$ bands in our SEDs because they are contaminated by strong emission from $\mathrm{H} \alpha$, the IR Ca II triplet, and $\mathrm{CO}$ bands, respectively. Our fits are not unique. We used the shape of the SED to set our input parameters. We used an inner radius $R_{\text {in }}$ of $10^{16} \mathrm{~cm}$ and an outer radius $R_{\text {out }}$ of $10^{17} \mathrm{~cm}$ as initial inputs, based on the optical and IR contributions to the SED. The input optical luminosity and temperature were based on the optical continuum.

At 126 days, the input SED was our best fit, indicating no dust was detected. Starting from 197 days but more clearly at 295 and 498 days, a small amount of dust $\left(\sim 7 \times 10^{-6} M_{\odot}\right.$ for carbon grains and $\sim 10^{-4} M_{\odot}$ for silicate grains) was required to fit the SED. The peak temperature of the dust is $\sim 500 \mathrm{~K}$. Figure 5 shows the SED evolution of SN 2017eaw, compared with SN 2004et (Maguire et al. 2010; Fabbri et al. 2011), with the best-fit models from MOCASSIN overplotted for each epoch. Solid and dashed lines represent amorphous carbon and

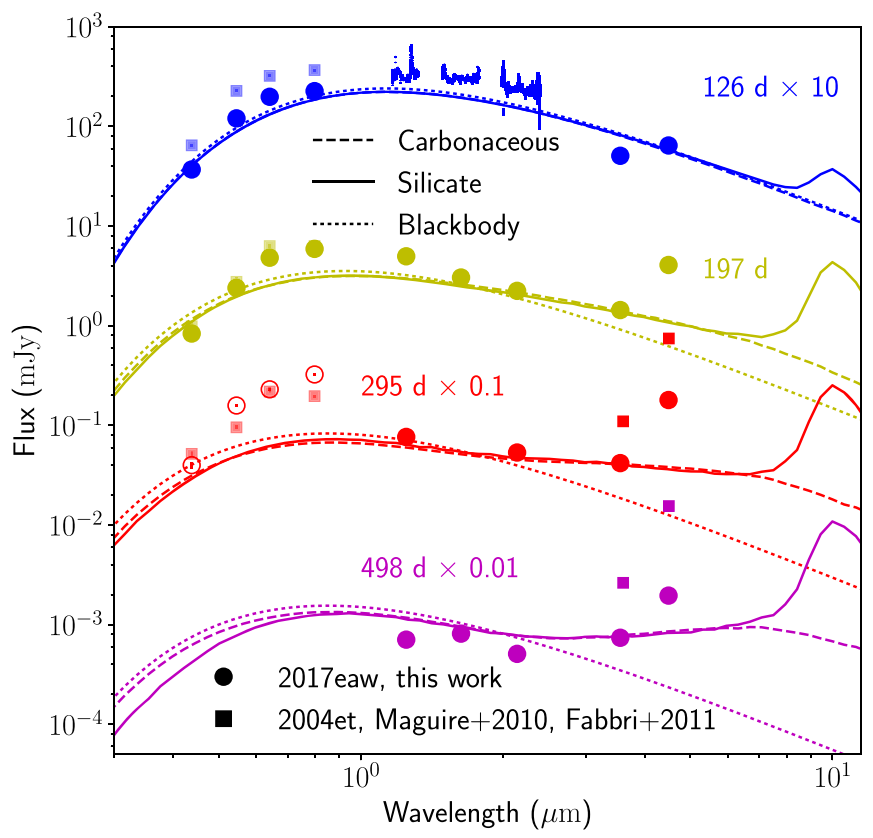

Figure 5. Evolution of the SED of SN 2017eaw (circles, optical data from Tsvetkov et al. 2018) in comparison to SN 2004et (squares, from Maguire et al. 2010) at 126, 197, 295, and 498 days. Optical data for the 295 days epoch are extrapolated and are shown with open symbols. SED models of a blackbody with dust grain emission from MOCASSIN are overplotted. Dashed lines represent a model with purely carbonaceous dust grains, while solid lines represent those with purely silicate grains. Dotted lines are blackbody fits, provided for comparison. The fits ignore the optical $R, I$ bands and Spitzer $4.5 \mu \mathrm{m}$ band due to contamination from the $\mathrm{H} \alpha$, Ca triplet, and $\mathrm{CO}$ fundamental bands, respectively.

silicate grains, respectively. Dotted lines are a blackbody provided for comparison. The excess flux in the $4.5 \mu \mathrm{m}$ band is similar in both SNe. For SN 2004et, it was shown to be due to emission from the CO fundamental band (Kotak et al. 2009). Our data cannot distinguish between carbonaceous and silicate dust, due to the lack of data around $10 \mu \mathrm{m}$. However, we note that in the case of SN 2004et, a broad silicate and $\mathrm{SiO}$ feature around $10 \mu \mathrm{m}$ was detected, pointing to some silicate grains (Kotak et al. 2009). Furthermore, dust condensation models predict that for SNe II-P, carbonaceous dust does not form until $\sim 1000$ days postexplosion (discussed above), while silicate dust (like forsterite) can start to form as early as 200 days (Figure 5 in Sarangi \& Cherchneff 2015). The comparison with SN 2004et and models suggests that the dust responsible for SN 2017eaw's $3.6 \mu \mathrm{m}$ excess at 295 days may be $\sim 10^{-4} M_{\odot}$ of silicates. In summary, the SED evolution of SN 2017eaw is similar to that of SN 2004et. A single-component blackbody can fit the SED reasonably well for the 126 and 197 days epochs, while a small amount of dust, likely $\sim 10^{-4} M_{\odot}$ of silicate dust, is required to fit the 295 and 498 days epochs. Finally we note that the rising IR excess is in agreement with the report by Rho et al. (2018) of rising flux in the red part of their $K$-band spectra starting at $\sim 120$ days.

\subsection{Spectroscopic Evolution and High-velocity Absorption Features}

Spectra of SN 2017eaw shown in Figure 4 evolved from the photospheric phase to the nebular phase after the 116 days 


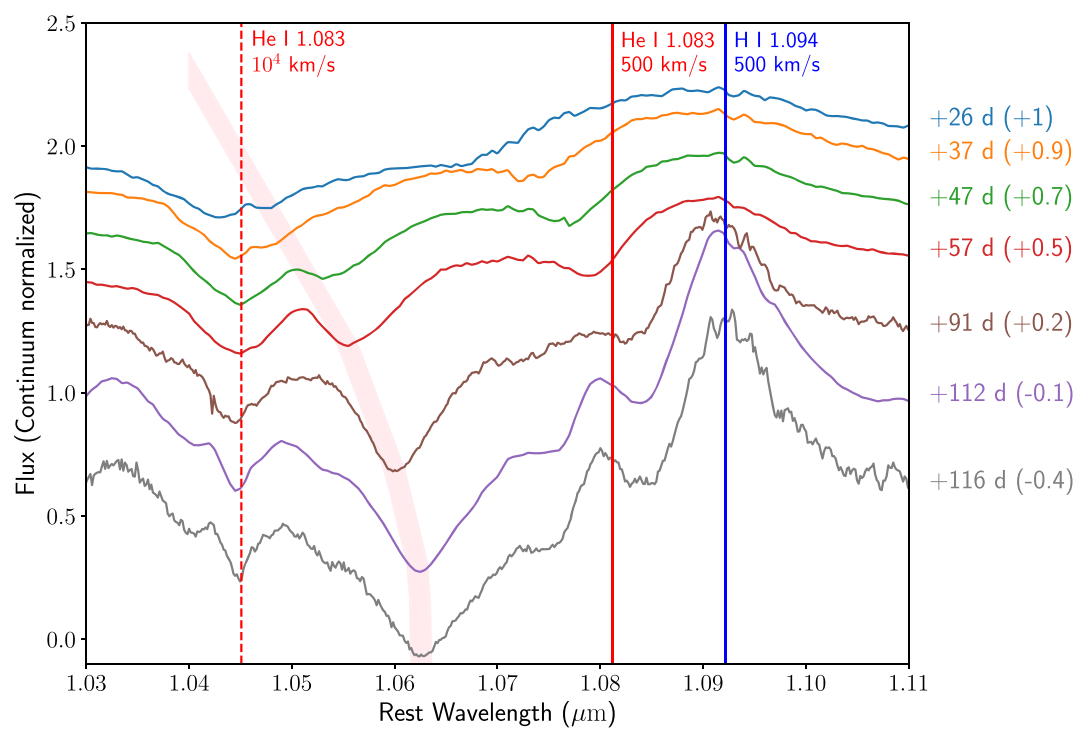

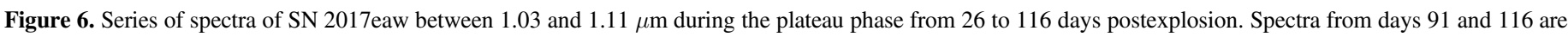

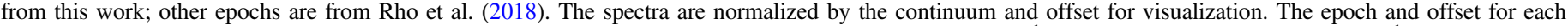

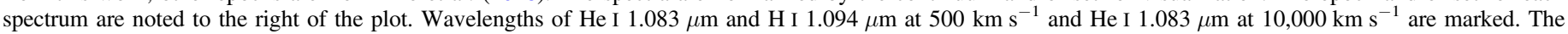
shaded band indicates the absorption trough, likely from the He I $1.083 \mu \mathrm{m}$ P-Cygni profile.

epoch. The photospheric spectra at 91 and 116 days were dominated by a strong continuum with hydrogen lines with P-Cygni profiles. Metal lines started to emerge from 116 days to 389 days as the $\mathrm{SN}$ transitioned into the nebular phase. The helium line at $1.083 \mu \mathrm{m}$ emerged at 116 days and became strong in the nebular phase, blended with $\mathrm{Pa}-\gamma$. Numerous metal species were also present in the nebular spectra, notably [Fe II] at $1.257,1.600$, and $1.644 \mu \mathrm{m}$. The latter two were blended with hydrogen. Na I at $2.206 \mu \mathrm{m}$ emerged at 116 days and became as strong as $\mathrm{Br}-\gamma$ in the last four epochs. Modeling these spectra to obtain physical parameters for the ejecta is outside the scope of this paper.

Figure 6 shows a series of continuum-normalized near-IR spectra of SN 2017eaw during the plateau phase from 26 to 116 days postexplosion. Data for days 91 and 116 are from this work, and the rest are from Rho et al. (2018). The hydrogen Paschen- $\gamma$ line at $1.094 \mu \mathrm{m}$ with a P-Cygni profile is present throughout the plateau phase with decreasing photospheric velocity, as expected in a normal SN II-P. Another P-Cygni absorption trough, likely from He I $1.083 \mu \mathrm{m}$, is present. It also has a decreasing velocity from $\sim 10^{4} \mathrm{~km} \mathrm{~s}^{-1}$ at 26 days to $\sim 5000 \mathrm{~km} \mathrm{~s}^{-1}$ at 116 days. The spectra show an absorption feature at $1.045 \mu \mathrm{m}$, corresponding to the $\mathrm{He}$ I $1.083 \mu \mathrm{m}$ line at $v=10,000 \mathrm{~km} \mathrm{~s}^{-1}$. While there is a S I $1.046 \mu \mathrm{m}$ line nearby, it is not expected to be present during the photospheric phase as the metal-rich ejecta have not yet been exposed. The velocity of this feature, unlike that of the P-Cygni absorption trough, remains constant throughout the photospheric phase. We identify this feature as the high-velocity He I $1.083 \mu \mathrm{m}$ absorption line, predicted by Chugai et al. (2007) in the case of CSM interactions. Chugai et al. (2007) argued that the highvelocity absorption features for $\mathrm{H} \alpha$ and $\mathrm{He}$ I $1.083 \mu \mathrm{m}$ are formed by the recombined gas in the outer ejecta excited by high-energy photons from the CSM interaction. The presence of this line provides evidence that there is CSM interaction going on in SN 2017eaw. We note here, however, that the highvelocity $\mathrm{He} \mathrm{I} 1.083 \mu \mathrm{m}$ absorption during the plateau is predicted as a result of a time-dependent ionization effect in the expanding ejecta (Dessart \& Hillier 2008).

\subsection{Temporal Evolution of the CO First Overtone Feature}

Our series of near-IR spectra capture the evolution of the 2.3-2.5 $\mu \mathrm{m}$ band of the CO vibrational first overtone $(\Delta v=2)$ transition. Rho et al. (2018) reported that the feature emerged at 124 days and strengthened until their last epoch at 205 days. Assuming an LTE level population, they inferred a growing CO mass from $(0.6-1.6) \times 10^{-4} M_{\odot}$ at 124 days to $(1.9-2.2) \times$ $10^{-4} M_{\odot}$ at 205 days, with the temperature declining in their last three epochs, reaching $2700 \mathrm{~K}$ at 205 days. Our spectrum at 389 days showed that the $\mathrm{CO}$ feature continued to strengthen and had become as prominent as hydrogen by this epoch. Spectra from 408,439 , and 480 days subsequently showed that the CO feature started to fade. The series of these last four spectra are shown in Figure 7 (left) in the linear scale to demonstrate the fading $\mathrm{CO}$ feature. Wavelengths for $\mathrm{CO}$ band heads of the $\Delta v=2$ transitions are overplotted, both for ${ }^{12} \mathrm{CO}$ and ${ }^{13} \mathrm{CO}$ with $v=-500 \mathrm{~km} \mathrm{~s}^{-1}$.

The fading of the $\mathrm{CO}$ first overtone feature may indicate either that $\mathrm{CO}$ molecules have been destroyed or that the gas has cooled down enough that the $v \geqslant 2$ vibrational levels are no longer excited. Some authors argued that $\mathrm{CO}$ can be easily destroyed by high-energy electrons from ${ }^{56} \mathrm{Co}$ decay and that most atomic carbon condenses into amorphous carbonaceous dust grains starting at 200-300 days postexplosion (Todini \& Ferrara 2001). However, in this scenario, the CO formation would have been inhibited in the first place (not formed and destroyed later), and their model predicted the $\mathrm{CO}$ mass for SN 1987A to be a factor of three smaller than what was observed. They did not address how dust grains could survive the same energetic electrons from ${ }^{56} \mathrm{Co}$ decay that destroyed the $\mathrm{CO}$ molecules.

Alternatively, the fading of the first overtone feature may indicate that the ejecta where $\mathrm{CO}$ formed have cooled enough that the $v \geqslant 2$ vibrational levels corresponding to the first overtone emission are no longer excited. The scenario is further supported by the fact that the $4.5 \mu \mathrm{m}$ excess in the SED (see Section 3.3), likely due to the CO fundamental emission, did not disappear. This is because the fundamental transition can 

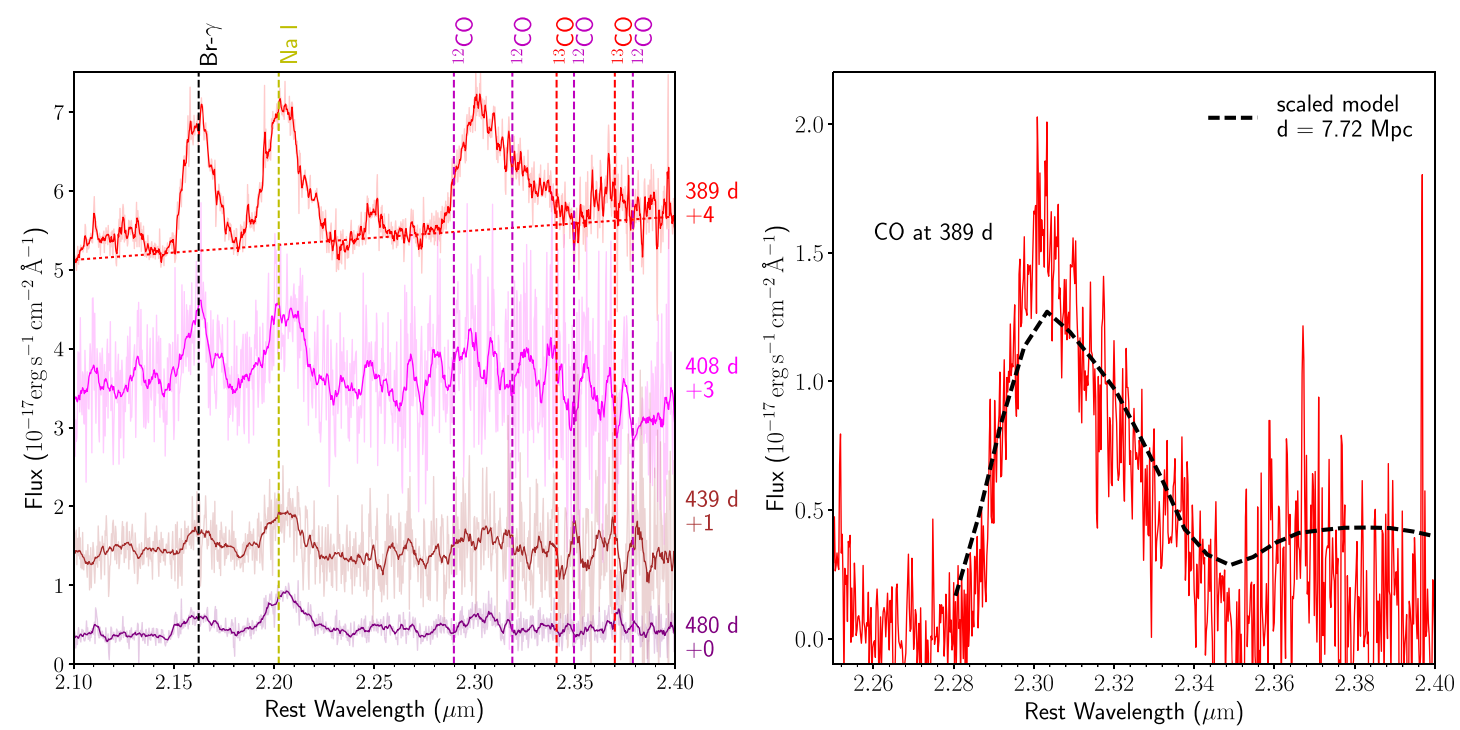

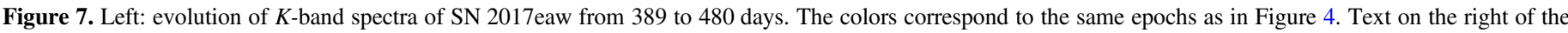

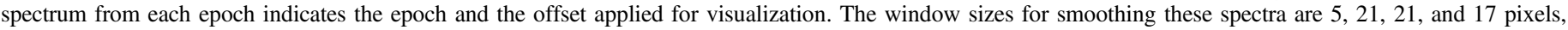

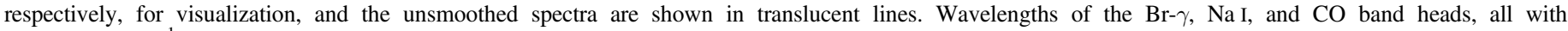

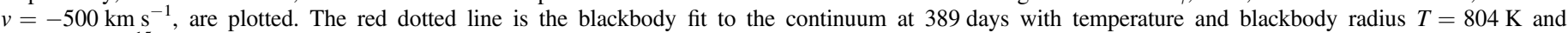

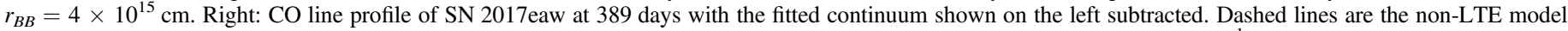

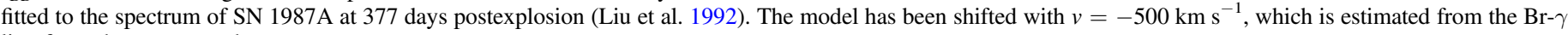
line from the same epoch.

happen as long as the $v=1$ level, which is less energetic, remains populated. Another line of evidence that $\mathrm{CO}$ did not get destroyed in SN 2017eaw's ejecta is the similarity between its CO line evolution and that of SN 1987A. Recall that in SN 2017eaw, the first overtone bands emerged at $\sim 200$ days and started to disappear at $\sim 400$ days, while the fundamental bands $(4.5 \mu \mathrm{m}$ band) remained detected at 566 days. This behavior is consistent with what was observed in SN 1987A, where the $\mathrm{CO}$ first overtone emerged as early as 100 days and faded by 574 days while the $\mathrm{CO}$ fundamental band remained strong until 600-700 days (e.g., Spyromilio et al. 1988; Meikle et al. 1989; Liu et al. 1992; Meikle et al. 1993). The survival of CO in SN ejecta was shown for SN 1987A by late-time observations with ALMA, more than $25 \mathrm{yr}$ postexplosion, that detected $\mathrm{CO}$ (along with $\mathrm{SiO}$ ) rotational emission (Kamenetzky et al. 2013; Abellán et al. 2017). In summary, the fading CO first overtone feature indicates that the ejecta have cooled, and not that $\mathrm{CO}$ molecules are destroyed.

The survival of $\mathrm{CO}$ is favored by more recent chemical evolution models that treat chemical reactions in $\mathrm{SN}$ ejecta more realistically (MNT, e.g., Sarangi \& Cherchneff 2013, 2015; Sluder et al. 2018). Specifically, the chemical evolution of the SN ejecta is modeled using a realistic network of chemical reactions to track molecules and dust formation together. Sarangi \& Cherchneff (2013, 2015), assuming stratified ejecta, found that in the ejecta layers where carbon is abundant (zones 4 and 5 in their papers), $\mathrm{CO}$ forms in the oxygen-rich layer (zone 4) and carbonaceous dust can only form in the oxygen-poor part (zone 5). This is because $\mathrm{CO}$ is more easily formed and is not destroyed once formed, so all carbon atoms in zone 4 end up in CO. Sluder et al. (2018) used a realistic ejecta model that has some radioactive species mixed into the ejecta to model molecule and dust dissociation due to the high-energy electrons from radioactive decays. They concluded that, while high-energy electrons keep the ejecta gas from becoming fully molecular, they do not destroy all $\mathrm{CO}$, and that up to $0.06 M_{\odot}$ of $\mathrm{CO}$ survives to 10,000 days (see their Figure 14). The implication of this result is that $\mathrm{CO}$ is not easily destroyed to form carbonaceous dust, as was concluded by Todini \& Ferrara (2001).

\subsection{Line Profile of the CO First Overtone Feature}

The CO emission from SN 2017eaw is similar to that from SN 1987A, not only in its temporal evolution, but also in the line profile. In this subsection, we consider our 389 days epoch, which has the strongest detection of the $\mathrm{CO}$ feature. To isolate SN 2017eaw's CO line profile, we first estimated and subtracted the underlying continuum by fitting a blackbody curve to parts of the $K$-band spectrum at 389 days without strong lines. The blackbody temperature and radius are $T=809 \mathrm{~K}$ and $r_{\mathrm{BB}}=4 \times 10^{15} \mathrm{~cm}$, respectively. The model is shown in Figure 7 (left). This warm continuum is likely coming from the same component that Rho et al. (2018) reported in their spectra. By 480 days, this continuum has substantially decreased. The continuum-subtracted $\mathrm{CO}$ profile is shown in Figure 7 (right).

The CO line profile of SN 2017eaw observed on day 389 is very similar to that of SN 1987A at 377 days (Spyromilio et al. 1988). On top of the continuum-subtracted CO line profile shown in Figure 7 (right), we plotted the non-LTE model by Liu et al. (1992), which was fitted to SN 1987A's spectrum at 377 days. We shifted the wavelength with $v=-500 \mathrm{~km} \mathrm{~s}^{-1}$ (blueshift), a line peak velocity estimated from the $\mathrm{Br}-\gamma$ line from the same epoch. We only scaled the flux of the model by the squared ratio of the distances to SNe 1987A $(51.4 \mathrm{kpc}$, Panagia 2005) and 2017eaw. The result assuming $d=$ $7.72 \mathrm{Mpc}$ fitted our data reasonably well. We next describe the Liu et al. (1992) model and how we can use it to explain SN 2017eaw.

The Liu et al. (1992) model predicts the CO emission features while accounting for non-LTE effects by assuming that the $\mathrm{CO}$ vibrational-level populations are determined by 
collisional excitation and radiative de-excitation. This is a reasonable assumption because, at low temperature and density at this epoch, the collisional timescale is an order of magnitude longer than the radiative timescale, rendering collisional deexcitation ineffective. Liu et al. (1992) contrasted their nonLTE results with the LTE results from Spyromilio et al. (1988) to demonstrate that the level population at this epoch is clearly non-LTE as their model provided a superior fit to the data (see their Figures 1 and 2 for LTE and non-LTE results, respectively). Further, the non-LTE models implied an order of magnitude higher CO mass for SN 1987A in comparison to LTE models. For the particular model of SN 1987A we use for comparison to our SN2017eaw data, the CO mass is $9.1 \times 10^{-4} M_{\odot}$, the temperature is $1800 \mathrm{~K}$, and the ejecta velocity is $2000 \mathrm{~km} \mathrm{~s}^{-1}$.

The fact that the model fitted to SN 1987A data at 377 days explains our SN2017eaw data well suggests that the CO properties (mass, temperature, and velocity) between the two SNe are similar. This implied CO mass of $\sim 10^{-3} M_{\odot}$ for SN 2017eaw is a factor of a few higher than the values determined by Rho et al. (2018); however, we note earlier that this discrepancy is expected from comparing non-LTE to LTE models. The line ratios between different transitions in this band are determined by the relative population in different vibrational excitation states. This is determined by the electron temperature since $\mathrm{CO}$ is excited by collisions with free electrons in this model (Liu et al. 1992). Comparing SN 2017eaw's CO profile to different models at $T=1800-4000 \mathrm{~K}$ from Liu et al. (1992), it is clear that the plotted model at $1800 \mathrm{~K}$ fits our data best. Finally, the width of the profile is determined by the ejecta velocity, and again, this model with $v=2000 \mathrm{~km} \mathrm{~s}^{-1}$ explains our data well. In summary, we are able to estimate electron temperature, CO mass, and velocity for SN 2017eaw at 389 days by comparing its observed $\mathrm{CO}$ first overtone line profile with a non-LTE model for SN 1987A's CO at 377 days. The similarity in line profiles indicates that the two SNe have similar CO mass, temperature, and velocity at this epoch, which is surprising since SN 1987A is a very different kind of explosion coming from a different type of progenitor (blue supergiant, instead of RSG). Future work fitting the entire sequence of SN 2017eaw spectra with non-LTE models is required to get a complete picture of its $\mathrm{CO}$ mass evolution.

\subsection{Molecule and Dust Formation in Comparison to Chemical Evolution Models}

Near-IR spectroscopy, along with cadenced Spitzer observations, provided a detailed monitoring of the chemical evolution of SN 2017eaw's ejecta, which can be compared to different chemical evolution models. The series of near-IR spectra presented by Rho et al. (2018) and this work are the best nebular-phase near-IR spectra of any SNe since SN 1987A. From previous subsections, we have presented the following. (1) CO formed in SN 2017eaw's ejecta by $\sim 200$ days (observed by Rho et al. 2018) and cooled such that the first overtone transitions were no longer excited by $\sim 400$ days. The $4.5 \mu \mathrm{m}$ band excess, likely due to the less energetic fundamental transitions, was still detected at 566 days, showing that the $\mathrm{CO}$ molecules were not destroyed. (2) A contribution of hot dust emission to the $3.6 \mu \mathrm{m}$ channel has been detected starting at 300 days postexplosion, indicating the presence of warm dust (which may be newly formed or preexisting). We estimated the dust mass of $7 \times 10^{-6} M_{\odot}$ and
$10^{-4} M_{\odot}$ assuming pure carbonaceous and silicate grains, respectively. We now compare these observations with predictions from the simpler CNT along with those from the more realistic MNT.

First, we consider the formation and survival of CO. CNT models generally consider molecule and dust formation as different processes. The only influence molecules have on dust formation is that they deplete different species in the gas phase available to form dust. For example, Todini \& Ferrara (2001) show, assuming a complete mixing of radioactive species throughout the ejecta, that most carbon atoms that end up in amorphous carbonaceous dust grains as $\mathrm{CO}$ molecules are destroyed by energetic electrons from ${ }^{56} \mathrm{Co}$ decays. MNT models, like Sarangi \& Cherchneff $(2013,2015)$, show that CO is not a precursor molecule to carbonaceous dust grain formation because $\mathrm{CO}$ and carbon dust form via different chemical pathways, in different parts of the ejecta. In their models, Sarangi \& Cherchneff (2015) assumed that the ejecta are stratified into different zones with different compositions. In the zones with abundant carbon, if oxygen is present, all carbon atoms are used up in CO. Carbonaceous grains, on the other hand, only form in the oxygen-poor part of the carbonrich ejecta. Sluder et al. (2018) presented results from their MNT models using a more realistic ejecta model with some degree of mixing. They showed that up to $10^{-2} M_{\odot}$ of $\mathrm{CO}$ can form in the first 100 days postexplosion in SN 1987A-like ejecta (massive progenitor) and survive until 10,000 days, where their simulation ends. The major role of $\mathrm{CO}$ in dust formation is that it radiatively cools the ejecta. $\mathrm{SiO}$ formation may also play a role here, but we do not have direct evidence, due to the lack of observations at wavelengths longer than $5 \mu \mathrm{m}$ (though we note that $\mathrm{SiO}$ was observed in SN 2004et). Our observations showing that $\sim 10^{-3} M_{\odot}$ of $\mathrm{CO}$ has formed by 389 days and that $\mathrm{CO}$ survives to at least 566 days agree with the scenario predicted by MNT.

Second, we consider the formation of dust and the evolution of its mass. Figure 8 shows the dust mass measurement of SN 2017eaw in comparison to some other SNe and model predictions from Todini \& Ferrara (2001), Sarangi \& Cherchneff (2015), and Sluder et al. (2018). CNT models tend to predict quick precipitation of dust in the $\mathrm{SN}$ ejecta, with Todini \& Ferrara (2001) predicting $0.1 M_{\odot}$ of amorphous carbon formed by 400 days and $0.4 M_{\odot}$ of $\mathrm{Mg}_{2} \mathrm{SiO}_{4}$ formed by 600 days. MNT, on the other hand, predicts a range of dust formation behavior depending on the progenitor mass and ejecta composition. For example, Sarangi \& Cherchneff (2015) predicted only $2 \times 10^{-2} M_{\odot}$ of $\mathrm{Mg}_{2} \mathrm{SiO}_{4}$ at 300 days with carbonaceous dust not forming until 900 days in their $15 M_{\odot}$ progenitor model with homogeneous $\mathrm{SN}$ ejecta and low $\left(0.01 M_{\odot}\right){ }^{56} \mathrm{Ni}$. Their $15 M_{\odot}$ progenitor model with a normal amount $\left(0.075 M_{\odot}\right)$ of ${ }^{56} \mathrm{Ni}$ predicts slower dust formation, with the total dust mass reaching $2 \times 10^{-2} M_{\odot}$ at around 800 days. For their $19 M_{\odot}$ models, they considered both homogeneous and clumpy ejecta. They found that the dust condenses gradually in the homogeneous ejecta, only reaching $10^{-2} M_{\odot}$ at 1100 days. In contrast, clumpy ejecta form $10^{-3} M_{\odot}$ of $\mathrm{Mg}_{2} \mathrm{SiO}_{4}$ at 100 days and reach $10^{-2} M_{\odot}$ by 300 days, with carbonaceous dust not forming until 700 days. In all cases, $\mathrm{Mg}_{2} \mathrm{SiO}_{4}$ dominates the total dust mass for the first 200-300 days of dust formation. In Sluder et al. (2018), another MNT model specifically for SN 1987A, which can be compared to the clumpy $19 M_{\odot}$ model of Sarangi \& Cherchneff 


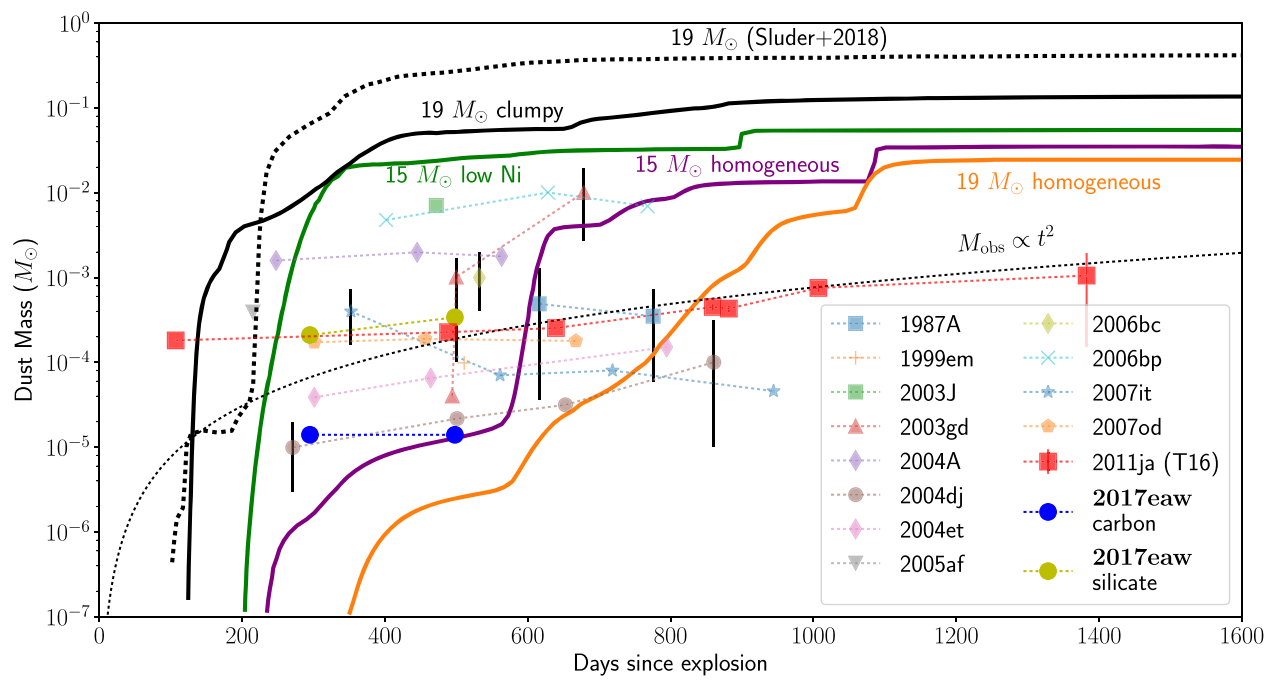

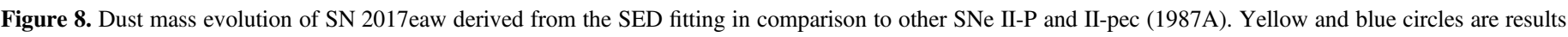

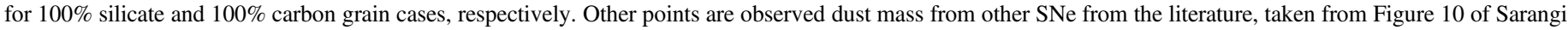

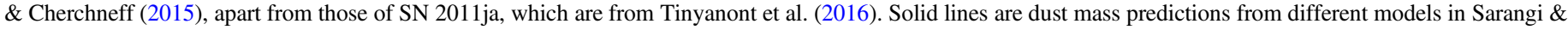

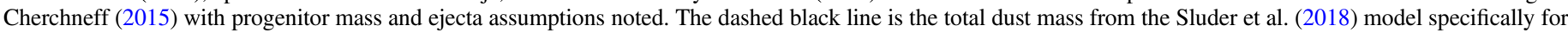

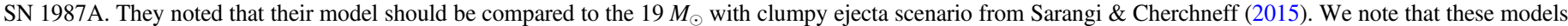

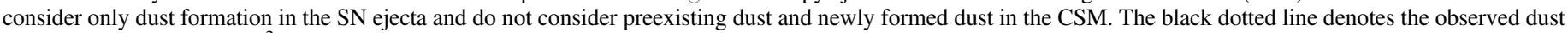
mass evolution of $M_{\mathrm{obs}} \propto t^{2}$ (arbitrarily scaled) expected from the evolution of the dust opacity (Dwek et al. 2018).

(2015), predicts $0.1 M_{\odot}$ of $\mathrm{Mg}_{2} \mathrm{SiO}_{4}$ by 300 days, with $10^{-2} M_{\odot}$ of carbonaceous dust by 500 days. We present this range of models to demonstrate that the predictions from different dust-formation models still widely disagree. Broadly, the dust mass inferred from the SED of SN 2017eaw of $10^{-4}$ $\left(10^{-6}\right) M_{\odot}$ assuming silicate (carbonaceous) dust, starting at 200 days, is a factor of 100 to 10,000 smaller than model predictions for a normal SN II-P.

The small amount of dust determined from the IR SED fitting at early time is in line with the dust mass determined from IR observations in other II-P SNe (e.g., Szalai et al. 2011; Tinyanont et al. 2016), including SNe 2004dj and 2004et (Kotak et al. 2009; Meikle et al. 2011). While the total amount of dust found at a few hundred days postexplosion is insufficient for SNe II-P to be a major source of cosmic dust production, observations of SN 1987A and Galactic SN remnants reveal $0.1-1 M_{\odot}$ of dust. As shown by ALMA observations, SN 1987A has as much as $0.2 M_{\odot}$ of dust in its inner ejecta in 2012, $26 \mathrm{yr}$ after the explosion (Indebetouw et al. 2014). Long-wavelength observations of supernova remnants also reveal similar amounts of dust surviving the passage of the reverse shock, available to be dispersed into the ISM. One explanation for this behavior is that dust continues to form in the ejecta over the course of a few years, as predicted by Sarangi \& Cherchneff (2015). Another possibility is that dust is formed quickly, but is optically thick at early time. As a result, the dust mass inferred from near to mid-IR observations at these epochs only accounts for dust in the outermost layer of the ejecta, then subsequently grows as $M_{\mathrm{obs}} \propto t^{2}$ (Dwek et al. 2018). From Figure 8, the dust mass we measured for SN 2017eaw (assuming silicate) evolves roughly consistently with this power law; however, long-term monitoring is required. We note here that in Dwek et al. (2018), the authors compared their $M_{\mathrm{obs}} \propto t^{2}$ prediction to the observed mass of SNe 1987A, 2004dj, and 2004et. In Figure 8, we show that the measured dust mass from SN 2011ja (Tinyanont et al. 2016) also follows this power law. Future observations, especially at very late times, of nearby CCSNe are still needed to probe the evolution of dust from a few hundred to a few thousand days postexplosion, which will test different chemical evolution models of SN ejecta. Such observations will be greatly enabled in the era of the James Webb Space Telescope (JWST).

\section{Summary and Conclusions}

In this paper, we have presented the following:

1. We presented observations of SN 2017eaw's progenitor in the $K s$ band from 344 to $\sim 1$ day before the explosion. We detected no photometric variability of the progenitor in this band down to $\Delta \nu L_{\nu} \lesssim 5000 L_{\odot}, 6 \%$ of the $K s$-band luminosity. Further, there was no evidence of short-term variability days before core collapse.

2. SN 2017eaw is similar in near-IR photometric evolution to normal SNe II-P 2002hh and 2004et, both also in NGC 6946, although SN 2017eaw is dimmer than SN 2004et by $0.5 \mathrm{mag}$ in the Spitzer/IRAC 3.6 and $4.5 \mu \mathrm{m}$ bands. The Spitzer/IRAC 3.6 and $4.5 \mu \mathrm{m}$ band light curves of SN 2017eaw and SN 2004et are nearly identical from the time of the explosion out to our last epoch at 566 days. Long-term monitoring of SN 2017eaw in the mid-IR will reveal whether it will have a late-time CSM interaction that rebrightens the mid-IR light curve, as was observed in SN 2004et.

3. The SED evolution showed a rising warm dust emission continuum in the $3.6 \mu \mathrm{m}$ band and an excess in the $4.5 \mu \mathrm{m}$ band that was likely due to CO fundamental band emission. Small amounts of dust $\left(10^{-6} M_{\odot}\right.$ carbonaceous or $10^{-4} M_{\odot}$ silicate) were needed to fit the SED at 197 and 295 days. While we cannot distinguish the two dust compositions from our data because of the lack of wavelength coverage around the silicate features at $\sim 10 \mu \mathrm{m}$, we note that silicate dust was detected in SN 2004et. We note that the small observed dust mass at 
early time may be due to the high opacity, even in the IR (Dwek et al. 2018).

4. We identify the absorption feature at $1.045 \mu \mathrm{m}$ as the $\mathrm{He} \mathrm{I}$ $1.083 \mu \mathrm{m}$ line at $10,000 \mathrm{~km} \mathrm{~s}^{-1}$. The presence of a highvelocity He I $1.083 \mu \mathrm{m}$ absorption line at the velocity near that of the shock front indicates that gas in the outer ejecta is excited by high-energy photons, suggestive of CSM interaction.

5. The $\mathrm{CO}$ first overtone band at $2.3 \mu \mathrm{m}$ continued to evolve from 205 days, as reported by Rho et al. (2018). The CO feature peaked at 389 days and subsequently faded with respect to the continuum in later epochs, similar to the behavior observed in SN 1987A. We conclude that the $\mathrm{CO}$ feature faded because the gas had cooled enough that $\mathrm{CO}$ vibrational levels required for the first overtone emission were no longer populated. The $4.5 \mu \mathrm{m}$ excess, likely due to the $\mathrm{CO}$ fundamental bands, was still detected at 566 days. The formation and survival of $\mathrm{CO}$ are in line with predictions from the MNT chemical evolution models presented by Sarangi \& Cherchneff (2013, 2015) and Sluder et al. (2018).

6. At 389 days, the $\mathrm{CO}$ line profile was similar to that of SN 1987A at 377 days. As a result, a non-LTE model fitted to SN 1987A (Liu et al. 1992) also fits our data. This suggests that the $\mathrm{CO}$ mass $\left(\sim 10^{-3} M_{\odot}\right)$ and temperature $(1800 \mathrm{~K})$ of the two $\mathrm{SNe}$ are similar at this epoch.

This study underlines the need for future IR observations of CCSNe in order to study molecule and dust formation in their ejecta in greater detail. Such observations, enabled by advances in IR instruments, especially JWST, will allow us to put constraints on chemical models of SNe ejecta and to paint a more complete picture of the contribution of CCSNe to the molecular and dust budget of the interstellar medium.

We thank Jim Fuller and Luc Dessart for helpful discussions and input on the paper draft. We also thank the anonymous referee for reviewing the article. Some of the data presented herein were obtained at the W. M. Keck Observatory, which is operated as a scientific partnership among the California Institute of Technology, the University of California, and the National Aeronautics and Space Administration. The Observatory was made possible by the generous financial support of the W. M. Keck Foundation. The authors wish to recognize and acknowledge the very significant cultural role and reverence that the summit of Maunakea has always had within the indigenous Hawaiian community. We are most fortunate to have the opportunity to conduct observations from this mountain. Some of the data presented herein were obtained at Palomar Observatory, which is operated by a collaboration between California Institute of Technology, Jet Propulsion Laboratory, Yale University, and National Astronomical Observatories of China. This work is based in part on observations made with the Spitzer Space Telescope, which is operated by the Jet Propulsion Laboratory, California Institute of Technology, under a contract with NASA. Support for this work was provided by NASA through an award issued by JPL/Caltech. This research has made use of the NASA/ IPAC Extragalactic Database (NED), which is operated by the Jet Propulsion Laboratory, California Institute of Technology, under contract with the National Aeronautics and Space
Administration. R.D.G. was supported by NASA and the United States Air Force.

Facilities: Hale (WIRC, TripleSpec), Spitzer, Keck (MOSFIRE, NIRES).

Software: astropy (Astropy Collaboration et al. 2013; PriceWhelan et al. 2018), spextool (Cushing et al. 2004), xtellcor (Vacca et al. 2003), MOSFIRE data reduction pipeline (McLean et al. 2012), MOCASSIN (v.2.02.72 Ercolano et al. 2003, 2005, 2008), Matplotlib (Hunter 2007).

\section{ORCID iDs}

Samaporn Tinyanont (iD https://orcid.org/0000-00021481-4676

Mansi M Kasliwal (i) https://orcid.org/0000-0002-5619-4938 Jeonghee Rho (iD https://orcid.org/0000-0003-3643-839X Jacob Jencson (iD https://orcid.org/0000-0001-5754-4007

Dimitri Mawet (iD https://orcid.org/0000-0002-8895-4735

Ricky Nilsson (iD https://orcid.org/0000-0002-5408-4954

Schuyler D Van Dyk (i) https://orcid.org/0000-00019038-9950

Ori D Fox (1) https://orcid.org/0000-0003-2238-1572

Geoffrey Clayton (i) https://orcid.org/0000-0002-0141-7436

\section{References}

Abellán, F. J., Indebetouw, R., Marcaide, J. M., et al. 2017, ApJL, 842, L24 Anand, G. S., Rizzi, L., \& Tully, R. B. 2018, AJ, 156, 105

Andrews, J. E., Gallagher, J. S., Clayton, G. C., et al. 2010, ApJ, 715, 541

Andrews, J. E., Krafton, K. M., Clayton, G. C., et al. 2016, MNRAS, 457, 3241

Argo, M., Torres, M. P., Beswick, R., \& Wrigley, N. 2017a, ATel, 10421

Argo, M., Torres, M. P., Beswick, R., \& Wrigley, N. 2017b, ATel, 10472

Arkharov, A. A., Klimanov, S. K., Mokrushina, A. A., Di Paola, A., \& Larionov, V. M. 2017, ATel, 10407

Astropy Collaboration, Robitaille, T. P., Tollerud, E. J., et al. 2013, A\&A, 558, A33

Barlow, M. J., Krause, O., Swinyard, B. M., et al. 2010, A\&A, 518, L138 Botticella, M. T., Trundle, C., Pastorello, A., et al. 2010, ApJL, 717, L52 Bright, J., Mooley, K., Fender, R., \& Horesh, A. 2017, ATel, 10394

Chakraborti, S., Ray, A., Smith, R., et al. 2013, ApJ, 774, 30 Chakraborti, S., Ray, A., Smith, R., et al. 2016, ApJ, 817, 22

Chugai, N. N., Chevalier, R. A., \& Utrobin, V. P. 2007, ApJ, 662, 1136 Cohen, M., Wheaton, W. A., \& Megeath, S. T. 2003, AJ, 126, 1090

Cushing, M. C., Vacca, W. D., \& Rayner, J. T. 2004, PASP, 116, 362

Dessart, L., \& Hillier, D. J. 2008, MNRAS, 383, 57

Dwek, E., Sarangi, A., \& Arendt, R. G. 2018, arXiv:1812.08234

Epinat, B., Amram, P., Marcelin, M., et al. 2008, MNRAS, 388, 500

Ercolano, B., Barlow, M. J., \& Storey, P. J. 2005, MNRAS, 362, 1038

Ercolano, B., Barlow, M. J., Storey, P. J., \& Liu, X.-W. 2003, MNRAS, 340,1136

Ercolano, B., Young, P. R., Drake, J. J., \& Raymond, J. C. 2008, ApJS, 175,534

Fabbri, J., Otsuka, M., Barlow, M. J., et al. 2011, MNRAS, 418, 1285

Fazio, G. G., Hora, J. L., Allen, L. E., et al. 2004, ApJS, 154, 10 Filippenko, A. V. 1997, ARA\&A, 35, 309

Foley, R. J., Smith, N., Ganeshalingam, M., et al. 2007, ApJL, 657, L105

Fox, O., Skrutskie, M. F., Chevalier, R. A., et al. 2009, ApJ, 691, 650

Fransson, C., Lundqvist, P., \& Chevalier, R. A. 1996, ApJ, 461, 993

Fuller, J. 2017, MNRAS, 470, 1642

Fuller, J., \& Ro, S. 2018, MNRAS, 476, 1853

Gall, C., Hjorth, J., Watson, D., et al. 2014, Natur, 511, 326

Gehrz, R. D., Roellig, T. L., Werner, M. W., et al. 2007, RScI, 78, 011302

Gerardy, C. L., Fesen, R. A., Nomoto, K., et al. 2002, PASJ, 54, 905

Gomez, H. L., Krause, O., Barlow, M. J., et al. 2012, ApJ, 760, 96

Grefensetette, B., Harrison, F., \& Brightman, M. 2017, ATel, 10427

Hanner, M. 1988, in Infrared Observations of Comets Halley and Wilson and Properties of the Grains, ed. M. S. Hanner (Washington, DC: NASA Sci. Tech. Info. Div.), 22

Harrison, F. A., Craig, W. W., Christensen, F. E., et al. 2013, ApJ, 770, 103 Helou, G., Kasliwal, M. M., Ofek, E. O., et al. 2013, ApJL, 778, L19 
Herter, T. L., Henderson, C. P., Wilson, J. C., et al. 2008, Proc. SPIE, 7014, $70140 \mathrm{X}$

Horne, K. 1986, PASP, 98, 609

Hosseinzadeh, G., Valenti, S., McCully, C., et al. 2018, ApJ, 861, 63

Houck, J. R., Roellig, T. L., van Cleve, J., et al. 2004, ApJS, 154, 18

Hunter, J. D. 2007, CSE, 9, 90

Indebetouw, R., Matsuura, M., Dwek, E., et al. 2014, ApJL, 782, L2

Johnson, S. A., Kochanek, C. S., \& Adams, S. M. 2018, MNRAS, 480, 1696

Kamenetzky, J., McCray, R., Indebetouw, R., et al. 2013, ApJL, 773, L34

Kasliwal, M. M., Bally, J., Masci, F., et al. 2017, ApJ, 839, 88

Khan, R. 2017, ATel, 10373

Kilpatrick, C. D., \& Foley, R. J. 2018, MNRAS, 481, 2536

Kochanek, C. S. 2018, MNRAS, 483, 3762

Kong, A. K. H., \& Li, K. L. 2017, ATel, 10380

Kotak, R., Meikle, P., van Dyk, S. D., Höflich, P. A., \& Mattila, S. 2005, ApJL, 628, L123

Kotak, R., Meikle, W. P. S., Farrah, D., et al. 2009, ApJ, 704, 306

Lau, R. M., Herter, T. L., Morris, M. R., Li, Z., \& Adams, J. D. 2015, Sci, 348,413

Le Bertre, T., Matthews, L. D., Gérard, E., \& Libert, Y. 2012, MNRAS, 422, 3433

Liu, W., Dalgarno, A., \& Lepp, S. 1992, ApJ, 396, 679

Mackey, J., Mohamed, S., Gvaramadze, V. V., et al. 2014, Natur, 512, 282

Maguire, K., Di Carlo, E., Smartt, S. J., et al. 2010, MNRAS, 404, 981

Mathis, J. S., Rumpl, W., \& Nordsieck, K. H. 1977, ApJ, 217, 425

Matsuura, M., Dwek, E., Barlow, M. J., et al. 2015, ApJ, 800, 50

Mauerhan, J. C., Smith, N., Filippenko, A. V., et al. 2013, MNRAS, 430, 1801

Mauerhan, J. C., Van Dyk, S. D., Johansson, J., et al. 2017, ApJ, 834, 118

McLean, I. S., Steidel, C. C., Epps, H. W., et al. 2012, Proc. SPIE, 8446, $84460 \mathrm{~J}$

Meikle, W. P. S., Allen, D. A., Spyromilio, J., \& Varani, G. F. 1989, MNRAS, 238, 193

Meikle, W. P. S., Kotak, R., Farrah, D., et al. 2011, ApJ, 732, 109

Meikle, W. P. S., Spyromilio, J., Allen, D. A., Varani, G.-F., \& Cumming, R. J. 1993, MNRAS, 261, 535

Milligan, S., Cranton, B. W., \& Skrutskie, M. F. 1996, Proc. SPIE, 2863, 2

Mooley, K. P., Cantwell, T., Titterington, D. J., et al. 2017, ATel, 10413

Moriya, T., Tominaga, N., Blinnikov, S. I., Baklanov, P. V., \& Sorokina, E. I. 2011, MNRAS, 415, 199

Nayana, A. J., \& Chandra, P. 2017a, ATel, 10388

Nayana, A. J., \& Chandra, P. 2017b, ATel, 10534
Ossenkopf, V., Henning, T., \& Mathis, J. S. 1992, A\&A, 261, 567

Panagia, N. 2005, in Cosmic Explosions, ed. J. M. Marcaide \& K. W. Weiler (Berlin: Springer), 585

Pastorello, A., Mattila, S., Zampieri, L., et al. 2008, MNRAS, 389, 113

Pooley, D., Lewin, W. H. G., Fox, D. W., et al. 2002, ApJ, 572, 932

Pozzo, M., Meikle, W. P. S., Rayner, J. T., et al. 2006, MNRAS, 368, 1169

Price-Whelan, A. M., Sipőcz, B. M., Günther, H. M., et al. 2018, AJ, 156, 123

Rho, J., Geballe, T. R., Banerjee, D. P. K., et al. 2018, ApJL, 864, L20

Rho, J., Kozasa, T., Reach, W. T., et al. 2008, ApJ, 673, 271

Rieke, G. H., Young, E. T., Engelbracht, C. W., et al. 2004, ApJS, 154, 25

Sarangi, A., \& Cherchneff, I. 2013, ApJ, 776, 107

Sarangi, A., \& Cherchneff, I. 2015, A\&A, 575, A95

Sarangi, A., Dwek, E., \& Arendt, R. G. 2018a, ApJ, 859, 66

Sarangi, A., Matsuura, M., \& Micelotta, E. R. 2018b, SSRv, 214, 63

Schlafly, E. F., \& Finkbeiner, D. P. 2011, ApJ, 737, 103

Schlegel, E. M. 1990, MNRAS, 244, 269

Skrutskie, M. F., Cutri, R. M., Stiening, R., et al. 2006, AJ, 131, 1163

Sluder, A., Milosavljević, M., \& Montgomery, M. H. 2018, MNRAS, 480,5580

Smartt, S. J. 2009, ARA\&A, 47, 63

Smith, N., \& Arnett, W. D. 2014, ApJ, 785, 82

Smith, N., Hinkle, K. H., \& Ryde, N. 2009a, AJ, 137, 3558

Smith, N., Silverman, J. M., Chornock, R., et al. 2009b, ApJ, 695, 1334

Spyromilio, J., Meikle, W. P. S., Learner, R. C. M., \& Allen, D. A. 1988, Natur, 334, 327

Szalai, T., \& Vinkó, J. 2013, A\&A, 549, A79

Szalai, T., Vinkó, J., Balog, Z., et al. 2011, A\&A, 527, A61

Szalai, T., Zsíros, S., Fox, O. D., Pejcha, O., \& Müller, T. 2018, arXiv:1803. 02571

Tinyanont, S., Kasliwal, M. M., Fox, O. D., et al. 2016, ApJ, 833, 231

Todini, P., \& Ferrara, A. 2001, MNRAS, 325, 726

Tomasella, L., Benetti, S., Cappellaro, E., et al. 2017, ATel, 10377

Tsvetkov, D. Y., Shugarov, S. Y., Volkov, I. M., et al. 2018, AstL, 44, 315

Vacca, W. D., Cushing, M. C., \& Rayner, J. T. 2003, PASP, 115, 389

van Dyk, S. D., Filippenko, A. V., Fox, O. D., et al. 2017, ATel, 10378

Werner, M. W., Roellig, T. L., Low, F. J., et al. 2004, ApJS, 154, 1

Wiggins, P. 2017, CBET, 4390

Wilson, J. C., Eikenberry, S. S., Henderson, C. P., et al. 2003, Proc. SPIE, 4841, 451

Woosley, S. E., \& Heger, A. 2015, ApJ, 810, 34

Yaron, O., Perley, D. A., Gal-Yam, A., et al. 2017, NatPh, 13, 510 